\title{
On the Capacity of the Intensity-Modulation Direct-Detection Optical Broadcast Channel
}

\author{
Anas Chaaban, Zouheir Rezki, and Mohamed-Slim Alouini
}

\begin{abstract}
The capacity of the intensity-modulation directdetection optical broadcast channel (OBC) is investigated, under both average and peak intensity constraints. An outer bound on the capacity region is derived by adapting Bergmans' approach to the OBC. Inner bounds are derived by using superposition coding with either truncated-Gaussian (TG) distributions or discrete distributions. While the discrete distribution achieves higher rates, the TG distribution leads to a simpler representation of the achievable rate region. At high signal-to-noise ratio (SNR), it is shown that the TG distribution is nearly optimal. It achieves the symmetric-capacity within a constant gap (independent of SNR), which approaches half a bit as the number of users grows. It also achieves the capacity region within a constant gap. At low SNR, it is shown that on-off keying (OOK) with time-division multipleaccess (TDMA) is optimal. This is interesting in practice since both OOK and TDMA have low complexity. At moderate SNR (typically $[0,8] \mathrm{dB}$ ), a discrete distribution with a small alphabet size achieves fairly good performance.
\end{abstract}

Index Terms-Intensity-modulation; optical broadcast; capacity region; truncated-Gaussian; discrete inputs.

\section{INTRODUCTION}

Optical wireless communication (OWC) refers to communication through an unguided medium using modulated light [2]-[4]. It has many advantages in comparison to RF, including higher data-rates and higher security/locality. Moreover, OWC has reduced electromagnetic interference in comparison to RF, which makes it an excellent candidate for communication in hospitals or airplanes, where electromagnetic compatibility is an essential requirement. Indoor OWC using visible-light is known as visible-light communications (VLC), and is the main topic of this paper.

For these reasons, interest in OWC has increased recently [5]-[10]. Focus has been towards studying the performance of OWC systems employing intensity modulation and direct detection (IM-DD), established by modulating light intensity at the source and using an intensity detector at the destination. Studying the performance of this simple implementation in terms of capacity is rather difficult. IM-DD systems are not captured by the intensively-studied Gaussian point-to-point (P2P) channel with a second-moment constraint [11]. The reason is that the transmit signal of an IM-DD system has to satisfy non-negativity, peak, and average constraints [9].

The authors are with King Abdullah University of Science and Technology (KAUST), Computer, Electrical and Mathematical Science and Engineering Division (CEMSE), Thuwal 23955-6900, Saudi Arabia. Email: \{anas.chaaban,zouheir.rezki, slim.alouini\}@kaust.edu.sa.

This work is supported in part by King Abdulaziz City of Science and Technology (KACST) under grant AT-34-145 and by the King Abdullah University of Science and Technology (KAUST).

Parts of this paper have been presented in the IEEE Globecom Workshop on Optical Wireless Communication, San Diego, CA, 2015 [1].
Although the capacity of the IM-DD P2P channel is still unknown in closed-form, some fairly tight bounds exist [8][10], and the high- and low-SNR capacity is known [9], [10].

Consider a scenario where an office light fixture is used to send information to multiple work-stations (users) using IM-DD for instance. The resulting system is an optical broadcast channel (OBC), and has been studied in [12]-[16]. In [12], the authors study the capacity of the Poisson broadcast channel. In [13]-[15], the authors analyze the performance of orthogonal code-division multiple-access. Orthogonalizing users this way allows serving multiple users in the OBC without interference. Hence, the channel from the transmitter to each receiver reduces to an IM-DD P2P, and capacity results on IM-DD P2P channels can be applied. However, as shown in [19], for a Gaussian broadcast channel which is physically degraded by nature, superposition coding (SC) is optimal and orthogonalizing users is not efficient. The performance of SC in the $\mathrm{OBC}$ in terms of bit-error rate and throughput has been recently studied in [16]. The MISO OBC was studied recently in [17], [18].

Thus, one should apply SC in the OBC. However, it is not clear which input distribution is optimal. Since the optimal distribution for the IM-DD P2P is unknown, finding the optimal distribution for the $\mathrm{OBC}$ is even more challenging. One expects the optimal distribution to be discrete as in the IM-DD P2P channel [20]. One could also use an exponential or a TG distribution [9], [10] to achieve good performance at high SNR. The advantage of those distributions is that their achievable rate can be expressed in a simpler form contrary to a discrete input distribution.

The goal of this paper is to study the capacity of the $\mathrm{N}$ user OBC, which models the downlink in VLC. The main focus is finding simple closed-form statements on the channel capacity. This requires developing outer and inner bounds on the capacity region of the channel. To this end, we modify Bergmans' outer bound [21] to obtain outer bounds on the capacity region of the OBC. Then, we develop inner bounds on the capacity region based on SC, where the source sends the sum of several symbols, each of which is desired by one user. To obtain the desired signal, assuming users are ordered in decreasing order of their received SNR, user $i$ decodes the signals intended to users $N, N-1, \cdots, i$ successively in this order, each time treating the remaining signals as noise. We derive the rate region achievable by $\mathrm{SC}$ with a TG distribution. We also use a discrete input distributions with finitely many uniformly spaced points [22] to develop an achievable rate region, where we use a successive optimization approach to optimize the distribution of the users. Then, we focus on three SNR regimes: high, low, and moderate. 
At high SNR, we provide an outer bound that can be written in a simple closed-form. Then, we study the symmetriccapacity at which we can transmit simultaneously to all users. We show that a TG distribution achieves the highSNR symmetric-capacity of the channel within a gap of 0.34 nats/transmission for the 2-user case. This gap is proportional to $\frac{1}{N} \log (N)$ for the $N$-user case. SC with a TG distribution is better than TDMA with the same distribution in general. We also show that the same distribution achieves the highSNR capacity region within a small gap. In particular, for an $N$-user $\mathrm{OBC}$, if the rate tuple $\left(R_{1}, R_{2}, \cdots, R_{N-1}, R_{N}\right)$ is inside our capacity outer bound, then the rate tuple $\left(R_{1}, R_{2}, \cdots, R_{N-1}, R_{N}-\delta\right)$ is achievable, where $\delta=0.68$ nats/transmission at most for $N=2$, and $\delta$ scales as $\frac{1}{2} \log (N)$ in the $N$-user case.

At low SNR, we show that coded OOK combined with TDMA is optimal as it achieves the channel's low-SNR capacity region, and hence also the low-SNR symmetriccapacity. The capacity region in this case is also given in a simple closed-form. At moderate SNR (typically $[0,8] \mathrm{dB}$ ), the discrete distribution achieves a larger rate region than TG. Furthermore, using a small number of mass points suffices to approach the symmetric-capacity. For instance, for a 2-user OBC with average and peak constraints $\mathcal{E}$ and $\mathcal{A}=2 \mathcal{E}$, and noise variances $\sigma_{1}^{2}$ and $\sigma_{2}^{2}=4 \sigma_{1}^{2}$ at users 1 and 2 , respectively, with $\frac{\varepsilon}{\sigma_{2}}=5 \mathrm{~dB}$, an input distribution with 4 symbols achieves a fair performance in terms of symmetric-rate.

The main contributions of the paper can thus be summarized as providing:

1) outer and inner bounds on the $\mathrm{OBC}$ capacity region,

2) the high-SNR capacity within a small constant gap, and

3) the low-SNR capacity.

Organization: The paper is organized as follows. We define the OBC formally in Sec. II. Then, for the 2-user case, we derive capacity outer bounds in Sec. III and inner bounds in Sec. IV. We focus on the high SNR and low/moderate SNR regimes in Sections V and VI, respectively. The results are extended to the $N$-user case in Sec. VII, and we conclude in Sec. VIII.

Notation: Throughout the paper, we use $g_{\mu, \nu}(x)$ to denote the Gaussian distribution with mean $\mu$ and variance $\nu^{2}$, and $G_{\mu, \nu}(x)$ to denote its CDF. We use normal-face fonts to denote scalars and bold-face fonts to denote vectors, and $\mathrm{CH}(\cdot)$ denote the convex hull of a set. We write $f(x) \stackrel{x \rightarrow \infty}{\longrightarrow} g(x)$ if $\lim _{x \rightarrow \infty}(f(x)-g(x))=0$ and we write $f(x) \stackrel{x \rightarrow 0}{\longrightarrow} g(x)$ if $\lim _{x \rightarrow 0} \frac{f(x)}{g(x)}=1$.

\section{The OpticAl BRoAdCAST CHANNEL}

Consider an $N$-user optical broadcast system, where information needs to be conveyed from a light source to users $i \in \mathcal{N}=\{1, \cdots, N\}$ using IM-DD (Fig. 1). The light intensity $X \geq 0$ has to satisfy peak and average constraints $X \leq \mathcal{A}$ and $\mathbb{E}[X] \leq \mathcal{E}$ due to safety and practical considerations. ${ }^{1}$ We denote the ratio $\frac{\varepsilon}{\mathcal{A}}$ by $\alpha \in[0,1]$.

\footnotetext{
${ }^{1}$ It might be further required that $\mathbb{E}[X]=\mathcal{E}$ to guarantee a desired lighting condition in an office environment for instance.
}

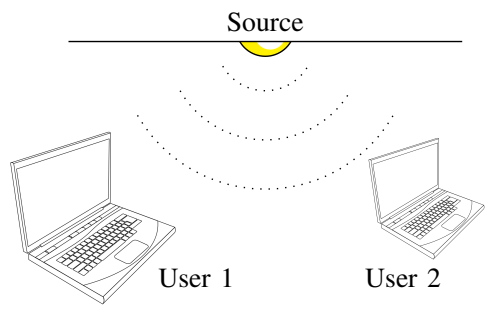

Fig. 1: An optical broadcast channel where light is used to send information to 2 users.

The received signal at user $i \in \mathcal{N}$ is $Y_{i}=X+Z_{i}$, where $Z_{i}$ represents the input-independent noise at user $i$, distributed according to $g_{0, \sigma_{i}}\left(z_{i}\right)$. Without loss of generality, $\sigma_{i}^{2} \geq \sigma_{i-1}^{2}$ for all $i \in \mathcal{N}$, where we formally define $\sigma_{0}^{2}=0$. We say that the resulting optical broadcast channel (OBC) has high SNR if $\mathcal{E} \gg \sigma_{N}$ and low SNR if $\mathcal{A} \ll \sigma_{1}$, which implies that both $\mathcal{A}$ and $\mathcal{E}$ are either much larger or smaller than $\sigma_{i}$ for all $i \in \mathcal{N}$.

The desired message at user $i$ is represented by a uniformly distributed random variable $W_{i} \in\left\{1, \cdots, 2^{n R_{i}}\right\}$ for some $n \in$ $\mathbb{N}$ and $R_{i} \geq 0$. The source encodes the independent messages $\left(W_{1}, \cdots, W_{N}\right)$ to a codeword $\boldsymbol{X} \in[0, \mathcal{A}]^{n}$ and sends this codeword. User $i$ receives $\boldsymbol{Y}_{i} \in \mathbb{R}^{n}$ and uses a decoder $\mathcal{D}_{i}$ to recover $W_{i}$. A rate tuple $\left(R_{1}, \cdots, R_{N}\right)$ is said to be achievable if there exists a coding scheme that satisfies $P_{e}=\operatorname{Prob}\left\{W_{i} \neq\right.$ $\left.\mathcal{D}_{i}\left(\boldsymbol{Y}_{i}\right), i \in \mathcal{N}\right\} \rightarrow 0$ as $n \rightarrow \infty$. The set of all achievable rate tuples is the capacity region of the channel, denoted $\mathrm{C}$.

Due to the symmetry of the noise distribution, using $\mathbb{E}[X]=$ $\mathcal{E}$ or $\mathbb{E}[X]=\mathcal{A}-\mathcal{E}$ achieves the same rate [9]. Thus, we restrict $\mathcal{E}$ to $\left(0, \frac{\mathcal{A}}{2}\right]$, i.e., $\alpha \in\left(0, \frac{1}{2}\right]$. Next, we focus on $N=2$. We generalize the results to $N \geq 2$ in Sec. VII.

\section{OUTER BOUND}

The OBC considered in this work belongs to the class of physically-degraded broadcast channels, for which the capacity is known [23]. The capacity region of a degraded broadcast channel satisfying the Markov chain $X \rightarrow Y_{1} \rightarrow Y_{2}$ is given by convex-hull of the closure of all rate pairs $\left(R_{1}, R_{2}\right) \in \mathbb{R}_{+}^{2}$ satisfying

$$
\begin{aligned}
& R_{2} \leq I\left(U ; Y_{2}\right), \\
& R_{1} \leq I\left(X ; Y_{1} \mid U\right),
\end{aligned}
$$

for some distribution $p(u) p(x \mid u) p\left(y_{1}, y_{2} \mid x\right)$ over $\mathcal{U} \times \mathcal{X} \times$ $y_{1} \times y_{2}$, where the cardinality of $\mathcal{U}$ is bounded by $|\mathcal{U}| \leq$ $\min \left\{|X|,\left|y_{1}\right|,\left|y_{2}\right|\right\}$, and where $X \in[0, \mathcal{A}]$ and $\mathbb{E}[X] \leq \mathcal{E}$. Specifying the optimal distribution $p(u) p(x \mid u)$ is a challenging problem in general. Our goal here is to obtain computable bounds which give better insights on the channel capacity. To compute such bounds, we need some upper bounds on the IM-DD P2P channel capacity, which are given next.

\section{A. Review of P2P Capacity Upper Bounds}

The next lemma reviews bounds on the IM-DD P2P channel capacity which were given in [9]. 


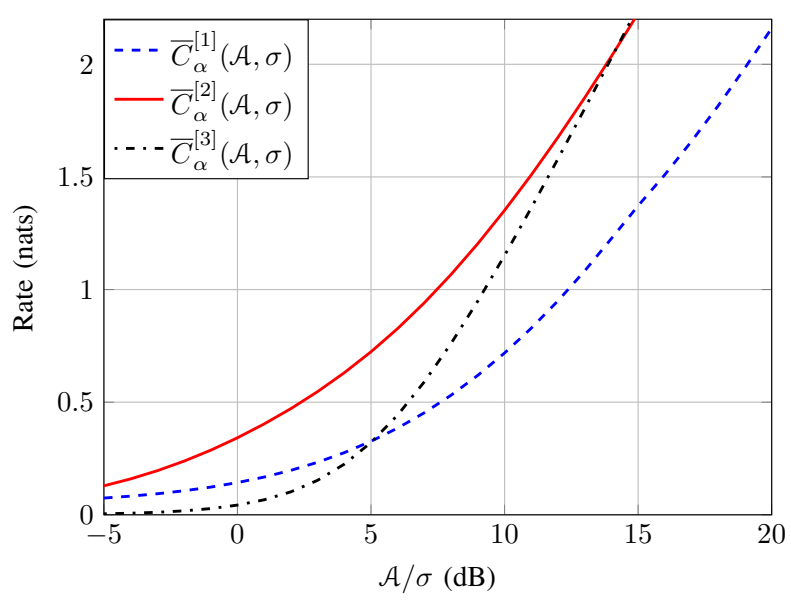

(a) $\alpha=1 / 10$.

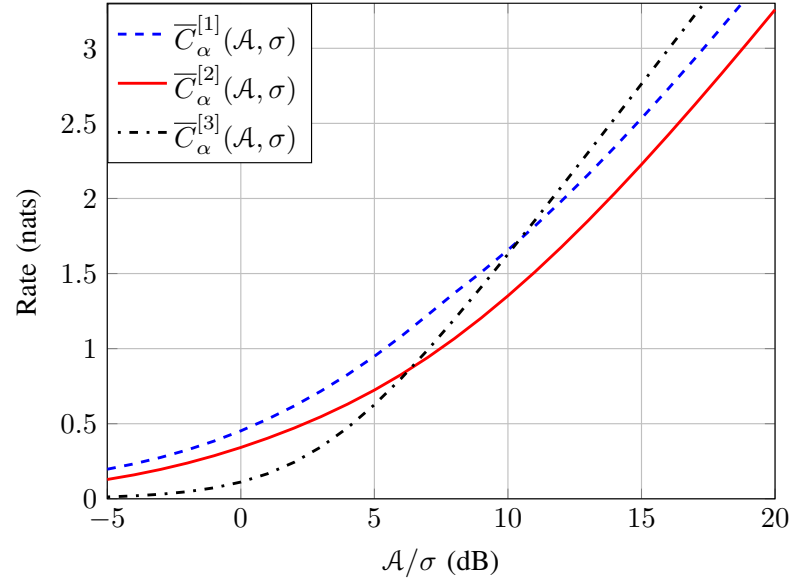

(b) $\alpha=1 / 2$.

Fig. 2: IM-DD P2P channel capacity upper bounds from [9]. None of the bounds uniformly dominates the others at all SNR.

Lemma 1 ( [9]): The capacity of the IM-DD P2P channel $Y=X+Z$ where $X \in[0, \mathcal{A}], \mathbb{E}[X] \leq \mathcal{E}=\alpha \mathcal{A}, \alpha \leq \frac{1}{2}$, and $Z \sim g_{0, \sigma}(z)$, is upper bounded by the following quantities

$$
\begin{aligned}
& \bar{C}_{\alpha}^{[1]}(\mathcal{A}, \sigma)=\inf _{\beta, \delta>0} B_{1}(\beta, \delta), \\
& \bar{C}_{\alpha}^{[2]}(\mathcal{A}, \sigma)=\inf _{\delta>0} B_{2}(\delta), \\
& \bar{C}_{\alpha}^{[3]}(\mathcal{A}, \sigma)=\frac{1}{2} \log \left(1+\frac{\alpha(1-\alpha) \mathcal{A}^{2}}{\sigma^{2}}\right),
\end{aligned}
$$

where $B_{1}(\beta, \delta)$ and $B_{2}(\delta)$ are given in (6) and (7) at the top of next page.

It is worth to note that none of those bounds uniformly dominates the others over the whole SNR range. Namely, $\bar{C}_{\alpha}^{[1]}(\mathcal{A}, \sigma)$ dominates at high SNR if $\alpha<1 / e, \bar{C}_{\alpha}^{[2]}(\mathcal{A}, \sigma)$ dominates at high SNR if $\alpha>1 / e$, and $\bar{C}_{\alpha}^{[3]}(\mathcal{A}, \sigma)$ dominates at low SNR (see Fig. 2). Thus, in the sequel, we will need all those three bounds.

The high-SNR asymptotic expressions of $\bar{C}_{\alpha}^{[1]}(\mathcal{A}, \sigma)$ and $\bar{C}_{\alpha}^{[2]}(\mathcal{A}, \sigma)$ will also be useful in the sequel. From [9], we have that $\bar{C}_{\alpha}^{[1]}(\mathcal{A}, \sigma)$ and $\bar{C}_{\alpha}^{[2]}(\mathcal{A}, \sigma)$ approach $\frac{1}{2} \log \left(1+\frac{e \alpha^{2} \mathcal{A}^{2}}{2 \pi \sigma^{2}}\right)$ and $\frac{1}{2} \log \left(1+\frac{\mathcal{A}^{2}}{2 \pi e \sigma^{2}}\right)$ respectively as $\frac{\varepsilon}{\sigma} \rightarrow \infty$. Those two bounds can be combined into the following high-SNR upper bound

$$
\begin{aligned}
\bar{C}_{\alpha}^{[h]}(\mathcal{A}, \sigma) & =\frac{1}{2} \log \left(1+\frac{c \mathcal{A}^{2}}{\sigma^{2}}\right), \\
c & =\min \left\{\frac{1}{2 \pi e}, \frac{e \alpha^{2}}{2 \pi}\right\} .
\end{aligned}
$$

Having the necessary ingredients, we can now derive an outer bound on the $\mathrm{OBC}$ capacity.

\section{B. Bergmans' Approach}

An outer bound on the $\mathrm{OBC}$ capacity region $\mathcal{C}$ can be derived by adapting Bergmans' approach [21]. For the Gaussian broadcast channel satisfying $\mathbb{E}\left[X^{2}\right] \leq P$ only, an important step in this approach involves using the fact that $h(X+Z) \leq$ $\frac{1}{2} \log \left(2 \pi e\left(\sigma^{2}+P\right)\right)$ when $Z \sim g_{0, \sigma}(z)$, with equality if $X \sim g_{0, \sqrt{P}}(x)$. Unfortunately, while the input distribution maximizing $h(X+Z)$ is known if $X \in \mathbb{R}$ and $\mathbb{E}\left[X^{2}\right] \leq P$, it is unknown when $X \in[0, \mathcal{A}]$ and $\mathbb{E}[X] \leq \mathcal{E}$ instead. To circumvent this problem, we use

$$
\begin{aligned}
h(X+Z) & =I(X ; X+Z)+h(Z) \\
& \leq \bar{C}_{\alpha}(\mathcal{A}, \sigma)+\log (\sqrt{2 \pi e} \sigma),
\end{aligned}
$$

where $\bar{C}_{\alpha}(\mathcal{A}, \sigma)$ is an upper bound on the IM-DD P2P channel capacity with the same constraints $\mathcal{A}$ and $\mathcal{E}$, and noise variance $\sigma^{2}$. The bound (10) is generalized to the vector case $h(\boldsymbol{X}+\boldsymbol{Z})$ next.

Lemma 2: For $X \in[0, \mathcal{A}]$ satisfying $\mathbb{E}[X] \leq \mathcal{E}$, and $Z \sim$ $g_{0, \sigma}(z)$, the entropy of the $n$-tuple $\boldsymbol{Y}=\boldsymbol{X}+\boldsymbol{Z}$ is upper bounded by $h(\boldsymbol{Y}) \leq n \bar{C}_{\alpha}^{[i]}(\mathcal{A}, \sigma)+n \log (\sqrt{2 \pi e} \sigma)$ for any $i \in\{1,2,3\}$.

Proof: By the subadditivity of entropy, we have $h(\boldsymbol{Y}) \leq$ $\sum_{i=1}^{n} h\left(Y_{i}\right)$ where $Y_{i}$ is the $i$-th component of $\boldsymbol{Y}$. Then, using (10) and Lemma 1 leads to the desired result.

Lemma 2 is useful in combination with Bergmans' approach to derive the following theorem.

Theorem 1: The capacity region $\mathcal{C}$ of the 2 -user $\mathrm{OBC}$ is outer bounded by $\overline{\mathcal{C}}^{[j]}=\bigcup_{\rho \in[0,1]} \overline{\mathcal{R}}^{[j]}(\rho)$ for any $j \in\{1,2,3\}$, where $\overline{\mathcal{R}}^{[j]}(\rho)$ is the set of $\left(R_{1}, R_{2}\right) \in \mathbb{R}_{+}^{2}$ that satisfy

$$
\begin{aligned}
& R_{1} \leq \frac{1}{2} \log \left(1+\frac{\sigma_{2}^{2}}{\sigma_{1}^{2}}\left(e^{2 \bar{C}_{\alpha}^{[j]}\left(\rho \mathcal{A}, \sigma_{2}\right)}-1\right)\right), \\
& R_{2} \leq \bar{C}_{\alpha}^{[j]}\left(\mathcal{A}, \sigma_{2}\right)-\bar{C}_{\alpha}^{[j]}\left(\rho \mathcal{A}, \sigma_{2}\right) .
\end{aligned}
$$

Proof: We have

$$
\begin{aligned}
& h\left(\boldsymbol{Y}_{2} \mid W_{2}\right) \geq h\left(\boldsymbol{Y}_{2} \mid W_{2}, \boldsymbol{X}\right)=h\left(\boldsymbol{Z}_{2}\right)=n \log \left(\sqrt{2 \pi e} \sigma_{2}\right), \\
& h\left(\boldsymbol{Y}_{2} \mid W_{2}\right) \leq h\left(\boldsymbol{Y}_{2}\right) \leq n \bar{C}_{\alpha}^{[j]}\left(\mathcal{A}, \sigma_{2}\right)+n \log \left(\sqrt{2 \pi e} \sigma_{2}\right),
\end{aligned}
$$

where the second line follows using Lemma 2 and since conditioning reduces entropy. Thus, since $\bar{C}_{\alpha}^{[j]}\left(\mathcal{A}, \sigma_{2}\right)$ is increasing 


$$
\begin{gathered}
B_{1}(\beta, \delta)=\log \left(\frac{\beta e^{-\frac{\delta^{2}}{2 \sigma^{2}}}}{\sqrt{2 \pi e} \sigma}+\frac{\mathcal{Q}\left(\frac{\delta}{\sigma}\right)}{\sqrt{e}}\right)+\frac{\mathcal{Q}\left(\frac{\delta}{\sigma}\right)}{2}+\frac{\left(\frac{\delta}{2 \sigma}+\frac{\sigma}{\beta}\right) e^{-\frac{\delta^{2}}{2 \sigma^{2}}}}{\sqrt{2 \pi}}+\frac{\delta^{2}\left(1-\mathcal{Q}\left(\frac{\delta+\mathcal{E}}{\sigma}\right)\right)}{2 \sigma^{2}}+\frac{\delta+\mathcal{E}}{\beta}, \\
B_{2}(\delta)=\left(1-2 \mathcal{Q}\left(\frac{2 \delta+\mathcal{A}}{2 \sigma}\right)\right) \log \left(\frac{\mathcal{A}+2 \delta}{\sigma \sqrt{2 \pi}\left(1-2 \mathcal{Q}\left(\frac{\delta}{\sigma}\right)\right)}\right)-\frac{1}{2}+\mathcal{Q}\left(\frac{\delta}{\sigma}\right)+\frac{\delta e^{-\frac{\delta^{2}}{2 \sigma^{2}}}}{\sqrt{2 \pi} \sigma}
\end{gathered}
$$

in $\mathcal{A}$ and approaches zero as $\mathcal{A} \rightarrow 0$, then we can write for some $\rho \in[0,1]$

$$
h\left(\boldsymbol{Y}_{2} \mid W_{2}\right)=n \bar{C}_{\alpha}^{[j]}\left(\rho \mathcal{A}, \sigma_{2}\right)+n \log \left(\sqrt{2 \pi e} \sigma_{2}\right) .
$$

To bound $R_{2}$, we write

$$
n\left(R_{2}-\varepsilon_{2 n}\right) \leq I\left(W_{2} ; \boldsymbol{Y}_{2}\right)=h\left(\boldsymbol{Y}_{2}\right)-h\left(\boldsymbol{Y}_{2} \mid W_{2}\right)
$$

using Fano's inequality with $\varepsilon_{2 n} \stackrel{n \rightarrow \infty}{\longrightarrow} 0$. By applying Lemma 2 and (13) we have

$$
h\left(\boldsymbol{Y}_{2}\right)-h\left(\boldsymbol{Y}_{2} \mid W_{2}\right) \leq n \bar{C}_{\alpha}^{[j]}\left(\mathcal{A}, \sigma_{2}\right)-n \bar{C}_{\alpha}^{[j]}\left(\rho \mathcal{A}, \sigma_{2}\right)
$$

Finally, by dividing by $n$ and letting $n \rightarrow \infty$, we get (12). To bound $R_{1}$, we write

$$
n\left(R_{1}-\varepsilon_{1 n}\right) \leq I\left(W_{1} ; \boldsymbol{Y}_{1}\right) \leq I\left(W_{1} ; \boldsymbol{Y}_{1} \mid W_{2}\right)
$$

using Fano's inequality with $\varepsilon_{1 n} \stackrel{n \rightarrow \infty}{\longrightarrow} 0$ and using $I\left(W_{1} ; \boldsymbol{Y}_{1}\right) \leq I\left(W_{1} ; \boldsymbol{Y}_{1}, W_{2}\right)=I\left(W_{1} ; \boldsymbol{Y}_{1} \mid W_{2}\right)+I\left(W_{1} ; W_{2}\right)$ with $I\left(W_{1} ; W_{2}\right)=0$ by the independence of $W_{1}$ and $W_{2}$. But $I\left(W_{1} ; \boldsymbol{Y}_{1} \mid W_{2}\right) \leq h\left(\boldsymbol{Y}_{1} \mid W_{2}\right)-h\left(\boldsymbol{Z}_{1}\right)$ since $h\left(\boldsymbol{Y}_{1} \mid W_{1}, W_{2}\right) \geq$ $h\left(\boldsymbol{Y}_{1} \mid W_{1}, W_{2}, \boldsymbol{X}\right)=h\left(\boldsymbol{Y}_{1} \mid \boldsymbol{X}\right)=h\left(\boldsymbol{Z}_{1}\right)$ due to the Markov chain $\left(W_{1}, W_{2}\right) \rightarrow \boldsymbol{X} \rightarrow \boldsymbol{Y}_{1}$ and the independence of $\boldsymbol{X}$ and $Z_{1}$. Thus,

$$
n\left(R_{1}-\varepsilon_{1 n}\right) \leq h\left(\boldsymbol{Y}_{1} \mid W_{2}\right)-h\left(\boldsymbol{Z}_{1}\right)
$$

Since $\sigma_{2}^{2} \geq \sigma_{1}^{2}$, and since the capacity of the broadcast channel depends only on the marginal distribution of $Y_{i}$ given $X$ [24], we write $Z_{2}=Z_{1}+\tilde{Z}_{2}$ where $\tilde{Z}_{2} \sim g_{0, \sqrt{\sigma_{2}^{2}-\sigma_{1}^{2}}}\left(\tilde{z}_{2}\right)$ independent of $Z_{1}$. Thus, $\boldsymbol{Y}_{2}=\boldsymbol{Y}_{1}+\tilde{Z}_{2}$. Using the conditional entropy-power inequality stating that $e^{\frac{2}{n} h\left(\boldsymbol{V}_{1}+\boldsymbol{V}_{2} \mid W\right)} \geq$ $e^{\frac{2}{n} h\left(\boldsymbol{V}_{1} \mid W\right)}+e^{\frac{2}{n} h\left(\boldsymbol{V}_{2} \mid W\right)}$ for conditionally independent $\boldsymbol{V}_{1}$ and $\boldsymbol{V}_{2}$, we write

$$
\begin{aligned}
h\left(\boldsymbol{Y}_{1} \mid W_{2}\right) & \leq \frac{n}{2} \log \left(e^{\frac{2}{n} h\left(\boldsymbol{Y}_{2} \mid W_{2}\right)}-e^{\frac{2}{n} h\left(\tilde{\boldsymbol{Z}}_{2} \mid W_{2}\right)}\right) \\
& =\frac{n}{2} \log \left(e^{\frac{2}{n} h\left(\boldsymbol{Y}_{2} \mid W_{2}\right)}-2 \pi e\left(\sigma_{2}^{2}-\sigma_{1}^{2}\right)\right) \\
& =\frac{n}{2} \log \left(2 \pi e \sigma_{1}^{2}+2 \pi e \sigma_{2}^{2}\left(e^{2 \bar{C}_{\alpha}^{[j]}\left(\rho \mathcal{A}, \sigma_{2}\right)}-1\right)\right),
\end{aligned}
$$

where the last step follows using (13). Plugging this inequality in (14), using $h\left(\boldsymbol{Z}_{1}\right)=n \log \left(\sqrt{2 \pi e} \sigma_{1}\right)$, dividing by $n$, and letting $n \rightarrow \infty$ yields (11). Finally, by taking the union over all $\rho \in[0,1]$, we get the statement of the theorem.

To assess the tightness of this bound, we derive capacity inner bounds in the next section.

\section{INNER BOUNDS}

An inner bound on $\mathcal{C}$ can be obtained using Cover's superposition coding (SC) technique [24, Theorem 1]. For $N=2$, this inner bound can be expressed as (1)-(2). To obtain the best inner bound, this region has to be maximized over input distributions $p(u, x)$ with $X \in[0, \mathcal{A}]$ and $\mathbb{E}[X] \leq \mathcal{E}$. Since the capacity achieving distribution of the IM-DD P2P channel is discrete [20], we expect the optimal $p(u, x)$ to be also discrete. Unfortunately, this leads to a rate region which is not computable in closed-form. We seek a simple computable rate region. To this end, we derive an inner bound based on truncated-Gaussian (TG) distributions, motivated by its simple rate expression and high SNR near-optimality for the IM-DD P2P channel [10].

\section{A. Truncated-Gaussian}

The TG distribution is described by the probability density function

$$
\tilde{g}(x)=\eta g_{\mu, \nu}(x) \text { for } x \in[0, \mathcal{A}]
$$

and $\tilde{g}(x)=0$ elsewhere, where $\eta=\left(G_{\mu, \nu}(\mathcal{A})-G_{\mu, \nu}(0)\right)^{-1}$. This distribution is completely characterized by the mean $\mu$ and the standard deviation $\nu$ of the entailed Gaussian distribution, and by its support $[0, \mathcal{A}]$. The parameter $\eta$ is a scaling factor required to ensure $\int \tilde{g}(x) d x=1$. The mean and variance of this distribution are respectively

$$
\begin{aligned}
\tilde{\mu} & =\nu^{2}(\tilde{g}(0)-\tilde{g}(\mathcal{A}))+\mu \\
\tilde{\nu}^{2} & =\nu^{2}(1-\mathcal{A} \tilde{g}(\mathcal{A})-\tilde{\mu}(\tilde{g}(0)-\tilde{g}(\mathcal{A}))) .
\end{aligned}
$$

In what follows, we call such a distribution a $(\mathcal{A}, \mu, \nu)$-TG distribution. Since we are dealing with a multi-user scenario, we will need multiple TG distributions with different $\mu, \nu$, and $\mathcal{A}$. For simplicity of notation, we will index these distributions by $i$. That is, for user $i$, we will use a $\left(\mathcal{A}_{i}, \mu_{i}, \nu_{i}\right)$-TG distribution with distribution function $\tilde{g}_{i}\left(x_{i}\right)$, where the induced scaling, mean, and variance are $\eta_{i}, \tilde{\mu}_{i}$, and $\tilde{\nu}_{i}^{2}$, respectively.

1) Achievable Rate Region: We first provide a general achievable rate region corresponding to this distribution, and then simplify it.

Definition 1: Let $\mathcal{P}$ be defined as

$$
\begin{aligned}
\mathcal{P}=\left\{\left(\boldsymbol{p}_{1}, \boldsymbol{p}_{2}\right) \mid \boldsymbol{p}_{i}\right. & =\left(\mathcal{A}_{i}, \mu_{i}, \nu_{i}\right) \in \mathbb{R}^{3}, \mathcal{A}_{i}, \nu_{i}>0 \\
& \left.i=1,2, \mathcal{A}_{1}+\mathcal{A}_{2}=\mathcal{A}, \tilde{\mu}_{1}+\tilde{\mu}_{2}=\mathcal{E}\right\} .
\end{aligned}
$$


Theorem 2: The region $\mathcal{R}_{T}\left(\boldsymbol{p}_{1}, \boldsymbol{p}_{2}\right)$ described by rate pairs $\left(R_{1}, R_{2}\right) \in \mathbb{R}_{+}^{2}$ satisfying

$$
\begin{aligned}
& R_{2} \leq \frac{1}{2} \log \left(\frac{\nu_{2}^{2}}{\tilde{\nu}_{2}^{2}}+\frac{\nu_{2}^{2}}{\tilde{\nu}_{1}^{2}+\sigma_{2}^{2}}\right)-\phi_{2}, \\
& R_{1} \leq \frac{1}{2} \log \left(\frac{\nu_{1}^{2}}{\tilde{\nu}_{1}^{2}}+\frac{\nu_{1}^{2}}{\sigma_{1}^{2}}\right)-\phi_{1},
\end{aligned}
$$

is achievable, where $\left(\boldsymbol{p}_{1}, \boldsymbol{p}_{2}\right) \in \mathcal{P}, \boldsymbol{p}_{i}=\left(\mathcal{A}_{i}, \mu_{i}, \nu_{i}\right), \phi_{i}=$ $\log \left(\eta_{i}\right)+\frac{1}{2}\left(\left(\mathcal{A}_{i}-\mu_{i}\right) \tilde{g}_{i}\left(\mathcal{A}_{i}\right)+\mu_{i} \tilde{g}_{i}(0)\right)$, and where $\tilde{g}_{i}\left(x_{i}\right)$ is the distribution function of a $\left(\mathcal{A}_{i}, \mu_{i}, \nu_{i}\right)$-TG random variable. The capacity region $\mathcal{C}$ of the 2 -user $\mathrm{OBC}$ thus satisfies $\mathcal{C} \supseteq$ $\underline{\mathcal{e}}_{T}=\bigcup_{\left(\boldsymbol{p}_{1}, \boldsymbol{p}_{2}\right) \in \mathcal{P}} \mathcal{R}_{T}\left(\boldsymbol{p}_{1}, \boldsymbol{p}_{2}\right)$.

Proof: The transmitter encodes the codewords $W_{1}$ and $W_{2}$ to two independent signals $\boldsymbol{X}_{1}$ and $\boldsymbol{X}_{2}$ so that the instances of $\boldsymbol{X}_{i}$ are i.i.d. according to a $\left(\mathcal{A}_{i}, \mu_{i}, \nu_{i}\right)$-TG distribution. The transmitter sends $\boldsymbol{X}=\boldsymbol{X}_{1}+\boldsymbol{X}_{2}$. Due to the average and peak constraints, we require $\mathcal{A}_{1}+\mathcal{A}_{2}=\mathcal{A}$ and $\tilde{\mu}_{1}+\tilde{\mu}_{2}=\mathcal{E}$. Both users decode $\boldsymbol{X}_{2}$, and user 1 decodes $\boldsymbol{X}_{1}$ after subtracting $\boldsymbol{X}_{2}$ from $\boldsymbol{Y}_{1}$. The achievable rates can be expressed as $R_{2} \leq I\left(X_{2} ; Y_{2}\right)$ and $R_{1} \leq I\left(X_{1} ; Y_{1} \mid X_{2}\right)$, which corresponds to choosing $U=X_{2}$ in (1)-(2). This inner bound can be computed numerically. An inner bound which is simpler to compute can be obtained as follows. Consider $I\left(X_{2} ; Y_{2}\right)$ first which can be written as $h\left(X_{2}\right)-h\left(X_{2} \mid Y_{2}\right)$. The entropy of $X_{2}$ is given by $h\left(X_{2}\right)=\frac{1}{2} \log \left(2 \pi e \nu_{2}^{2}\right)-\phi_{2}$. On the other hand, $h\left(X_{2} \mid Y_{2}\right)$ can be upper bounded by $h\left(\bar{X}_{2} \mid \bar{Y}_{2}\right)$ where $\left(\bar{X}_{2}, \bar{Y}_{2}\right)$ is jointly Gaussian with covariance matrix $\left(\begin{array}{ll}\tilde{\nu}_{2}^{2} & \tilde{\nu}_{2}^{2} \\ \tilde{\nu}_{2}^{2} & \tilde{\nu}_{1}^{2}+\tilde{\nu}_{2}^{2}+\sigma_{2}^{2}\end{array}\right)$, since the Gaussian distribution maximizes conditional differential entropy under a covariance constraint [25]. Thus, $h\left(X_{2} \mid Y_{2}\right) \leq \frac{1}{2} \log \left(2 \pi e_{\frac{\tilde{\nu}_{2}^{2}}{\left.\tilde{\nu}_{1}^{2}+\tilde{\nu}_{2}^{2}+\sigma_{2}^{2}\right)}}^{2}\right)$. Therefore, $I\left(X_{2} ; Y_{2}\right) \geq \frac{1}{2} \log \left(\frac{\nu_{2}^{2}}{\tilde{\nu}_{2}^{2}}+\frac{\nu_{2}^{2}}{\tilde{\nu}_{1}^{2}+\sigma_{2}^{2}}\right)-\phi_{2}$ and we get (18). Similarly, we can show that $I\left(X_{1} ; Y_{1} \mid X_{2}\right) \geq \frac{1}{2} \log \left(\frac{\nu_{1}^{2}}{\widetilde{\nu}_{1}^{2}}+\frac{\nu_{1}^{2}}{\sigma_{1}^{2}}\right)-\phi_{1}$ leading to (19). This proves the achievability of $\mathcal{R}_{T}\left(\boldsymbol{p}_{1}, \boldsymbol{p}_{2}\right)$. By taking the union over $\mathcal{P}$, we obtain the capacity region inner bound $\underline{\mathfrak{e}}_{T}$.

Since the optimal $\left(\boldsymbol{p}_{1}, \boldsymbol{p}_{2}\right)$ is difficult to find, we propose $\boldsymbol{p}_{1}$ and $\boldsymbol{p}_{2}$ which lead to a simpler inner bound, within a constant gap of the outer bound $\overline{\mathcal{C}}$ at high SNR.

2) Achievable Region Simplification: Note that if we choose $\mu \in[0, \mathcal{A}]$ and $\nu=\frac{1}{3} \min \{\mu, \mathcal{A}-\mu\}$, the $(\mathcal{A}, \mu, \nu)$-TG distribution becomes almost identical to a Gaussian distribution $g_{\mu, \nu}(x)$, since in this case, $\eta$ is close to 1 . We use this to simplify $\mathcal{R}\left(\boldsymbol{p}_{1}, \boldsymbol{p}_{2}\right)$ as follows.

Proposition 1: Let $\mathcal{R}_{T}^{\prime}(\beta), \beta \in[0,1]$, be the set of rate pairs $\left(R_{1}, R_{2}\right) \in \mathbb{R}_{+}^{2}$ satisfying

$$
\begin{aligned}
& R_{2} \leq \frac{1}{2} \log \left(1+\frac{\alpha^{2}(1-\beta)^{2} \mathcal{A}^{2}}{\left(\alpha^{2} \beta^{2} \mathcal{A}^{2}+9 \sigma_{2}^{2}\right)\left(1+\epsilon_{\mu}\right)^{2}}\right)-\epsilon_{\phi}, \\
& R_{1} \leq \frac{1}{2} \log \left(1+\frac{\alpha^{2} \beta^{2} \mathcal{A}^{2}}{9 \sigma_{1}^{2}\left(1+\epsilon_{\mu}\right)^{2}}\right)-\epsilon_{\phi},
\end{aligned}
$$

with $\epsilon_{\phi}=0.016$ and $\epsilon_{\mu}=0.0015$. Then, the rate region $\underline{\mathrm{e}}_{T}^{\prime}=\bigcup_{\beta \in[0,1]} \mathcal{R}_{T}^{\prime}(\beta)$ is achievable.

Proof: First, we choose $\mathcal{A}_{1}=\beta \mathcal{A}$ and $\mathcal{A}_{2}=(1-\beta) \mathcal{A}$, and we fix $\tilde{\mu}_{i}=\alpha \mathcal{A}_{i}$. This guarantees $\tilde{\mu}_{1}+\tilde{\mu}_{2}=\mathcal{E}$. Since $\alpha \leq$ $\frac{1}{2}$, then $\mu_{i} \leq \tilde{\mu}_{i} \leq \frac{\mathcal{A}_{i}}{2}, i=1,2$. Then we choose $\nu_{i}=\frac{\mu_{i}}{3}$ with $\mu_{i} \geq 0$. This choice leads to $\phi_{i} \leq \epsilon_{\phi}, \quad \mu_{i} \leq \tilde{\mu}_{i}<\mu_{i}\left(1+\epsilon_{\mu}\right)$, and $\left(1-\epsilon_{\nu}\right) \nu_{i}^{2} \leq \tilde{\nu}_{i}^{2} \leq \nu_{i}^{2}$, where $\epsilon_{\phi}=0.016, \epsilon_{\mu}=0.0015$, and $\epsilon_{\nu}=0.0267$ (cf. Appendix A). Thus we can write

$$
\begin{aligned}
\frac{1}{2} \log \left(\frac{\nu_{2}^{2}}{\tilde{\nu}_{2}^{2}}+\frac{\nu_{2}^{2}}{\tilde{\nu}_{1}^{2}+\sigma_{2}^{2}}\right)-\phi_{2} & \geq \frac{1}{2} \log \left(1+\frac{\nu_{2}^{2}}{\nu_{1}^{2}+\sigma_{2}^{2}}\right)-\epsilon_{\phi} \\
\frac{1}{2} \log \left(\frac{\nu_{1}^{2}}{\tilde{\nu}_{1}^{2}}+\frac{\nu_{1}^{2}}{\sigma_{1}^{2}}\right)-\phi_{1} & \geq \frac{1}{2} \log \left(1+\frac{\nu_{1}^{2}}{\sigma_{1}^{2}}\right)-\epsilon_{\phi} .
\end{aligned}
$$

Since $\tilde{\mu}_{1}=\alpha \beta \mathcal{A}, \nu_{1}=\frac{\mu_{1}}{3}$, and $\mu_{1} \leq \tilde{\mu}_{1} \leq \mu_{1}\left(1+\epsilon_{\mu}\right)$, we have $\frac{\alpha \beta \mathcal{A}}{3\left(1+\epsilon_{\mu}\right)} \leq \nu_{1} \leq \frac{\alpha \beta \mathcal{A}}{3}$, and thus, $\nu_{1}^{2} \geq \frac{(\alpha \beta \mathcal{A})^{2}}{9\left(1+\epsilon_{\mu}\right)^{2}}$. Similarly, we have $\frac{\nu_{2}^{2}}{\nu_{1}^{2}+\sigma_{2}^{2}} \geq \frac{(\alpha(1-\beta) \mathcal{A})^{2}}{\left(\alpha^{2} \beta^{2} \mathcal{A}^{2}+9 \sigma_{2}^{2}\right)\left(1+\epsilon_{\mu}\right)^{2}}$ which concludes the proof.

Since $\epsilon_{\mu}$ and $\epsilon_{\phi}$ are small, they will be neglected henceforth. While this inner bound is simple and fairly tight at high SNR, it is not at moderate/low SNR. Next, we provide another inner bound using a discrete input distribution in the spirit of [22].

\section{B. Discrete Input}

Similar to [22], we consider distributions of the form

$$
p_{X_{i}}\left(x_{i}\right)=\sum_{k=0}^{K_{i}} a_{i k} \delta\left(x-k \ell_{i}\right), \quad i=1,2,
$$

where $\delta(\cdot)$ is the Dirac delta, for some $K_{i}$ and $\ell_{i}$ such that $K_{i} \ell_{i}=\mathcal{A}_{i}, \mathbb{E}\left[X_{i}\right]=\mathcal{E}_{i}, \mathcal{A}_{1}+\mathcal{A}_{2}=\mathcal{A}$, and $\mathcal{E}_{1}+\mathcal{E}_{2}=\mathcal{E}$. The transmitter sends $X=X_{1}+X_{2}$. Note that if $K+1$ is the number of mass points of $X$, then $K_{1}+K_{2}+1 \leq$ $K+1 \leq\left(K_{1}+1\right)\left(K_{2}+1\right)$, where the lower bound corresponds to the case $\ell_{1}=\ell_{2}$, and the upper bound to the case where $k_{1} \ell_{1} \neq k_{2} \ell_{2}$ for all $k_{i} \in\left\{1, \cdots, K_{i}\right\}, i=1,2$. For simplicity, we focus on the second case.

1) Sequential Distribution Optimization: To derive distributions $p_{X_{1}}$ and $p_{X_{2}}$ which achieve good performance, we use a sequential approach. In this approach, we first find the distribution $p_{X_{1}}$ which maximizes $H\left(X_{1}\right)$ for a given $K_{1}$. This problem has been solved in [22]. Then, given $H\left(X_{1}\right)$, we find the distribution $p_{X_{2}}$ which maximizes $H\left(X_{1}+X_{2}\right)$ for a given $K_{2}$. This optimization determines the probabilities $a_{i k}, i \in\{1,2\}, k \in\left\{0, \cdots, K_{i}\right\}$, and allows us to evaluate the achievable rates $R_{1}=I\left(X_{1} ; Y_{1} \mid X_{2}\right)$ and $R_{2}=I\left(X_{2} ; Y_{2}\right)$.

We use this approach for the following reason. The achievable rate of user 1 is given by $R_{1}=I\left(X_{1} ; Y_{1} \mid X_{2}\right)=h\left(X_{1}+\right.$ $\left.Z_{1}\right)-h\left(Z_{1}\right)$. The noise entropy is $h\left(Z_{1}\right)=\log \left(\sqrt{2 \pi e} \sigma_{1}\right)$, and $h\left(X_{1}+Z_{1}\right) \geq \frac{1}{2} \log \left(e^{2 H\left(X_{1}\right)}+2 \pi e \sigma_{1}^{2}\right)$ by the entropy power inequality (EPI). Thus, $R_{1} \geq \frac{1}{2} \log \left(\frac{e^{2 H\left(X_{1}\right)}}{2 \pi e \sigma_{1}^{2}}+1\right)$. To maximize this rate, instead of maximizing $I\left(X_{1} ; Y_{1} \mid X_{2}\right)$ which is a difficult problem, one can resort to maximizing $H\left(X_{1}\right)$ instead. This does not necessarily maximize $I\left(X_{1} ; Y_{1} \mid X_{2}\right)$, but leads to a fairly good performance [22].

Given $H\left(X_{1}\right)$, a similar argument can be applied to $I\left(X_{2} ; Y_{2}\right)$. We have $R_{2}=I\left(X_{2} ; Y_{2}\right)=h\left(X_{1}+X_{2}+\right.$ $\left.Z_{2}\right)-h\left(X_{1}+Z_{2}\right)$. For a given $p_{X_{1}}$, the last term is fixed. The first term can be lower bounded by $h\left(X_{1}+X_{2}+\right.$ $\left.Z_{2}\right) \geq \frac{1}{2} \log \left(e^{2 H\left(X_{1}+X_{2}\right)}+2 \pi e \sigma_{2}^{2}\right)$ using the EPI. This lower bound can be maximized by finding $p_{X_{2}}$ which maximizes $H\left(X_{1}+X_{2}\right)$ given $p_{X_{1}}$. Due the imposed condition $k_{1} \ell_{1} \neq$ $k_{2} \ell_{2} \forall k_{i} \in\left\{1, \cdots, K_{i}\right\}$, the mapping from $\left(X_{1}, X_{2}\right)$ to 


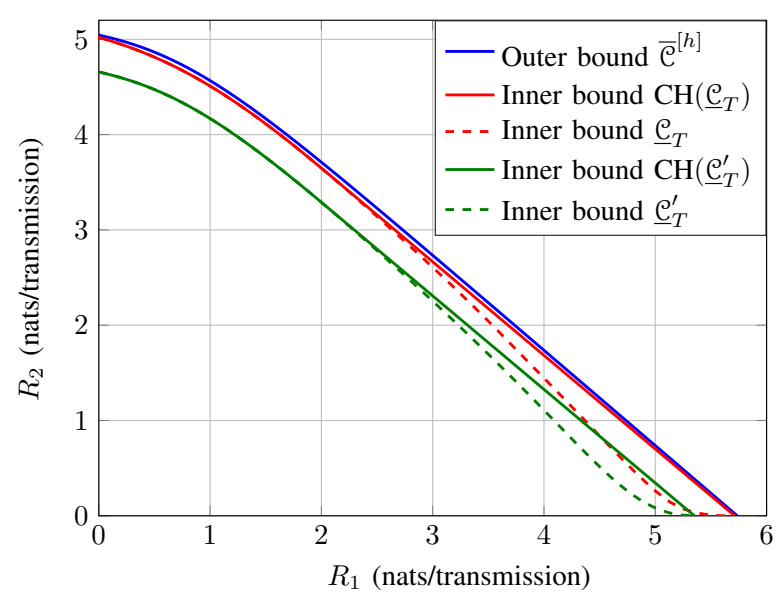

(a) $\alpha=\frac{1}{2}$.

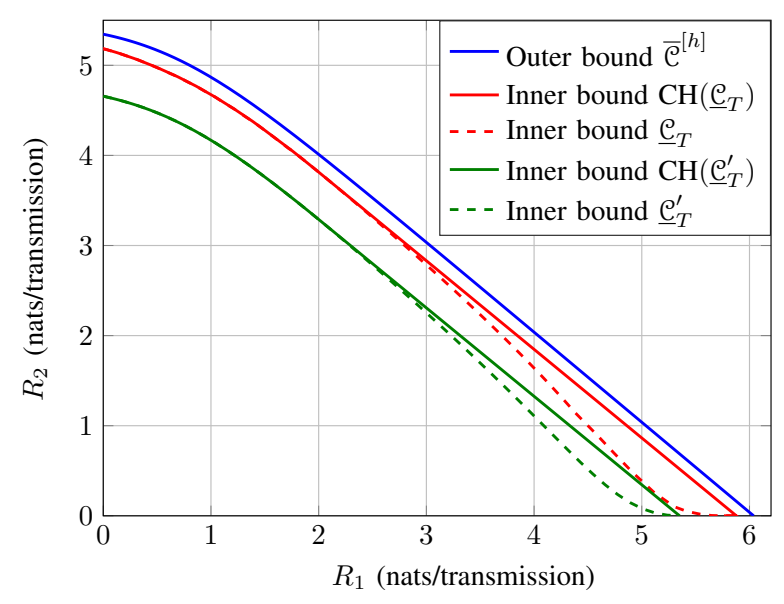

(b) $\alpha=\frac{1}{5}$.

Fig. 3: Capacity region outer and inner bounds for an $\mathrm{OBC}$ with $\frac{\varepsilon}{\sigma_{2}}=25 \mathrm{~dB}$ and $\sigma_{2}=2 \sigma_{1}$.

$X=X_{1}+X_{2}$ is one-to-one, and thus $H\left(X_{1} \mid X\right)=0$. Hence, $H(X)=H\left(X, X_{1}\right)-H\left(X_{1} \mid X\right)=H\left(X, X_{1}\right)=$ $H\left(X_{1}\right)+H\left(X_{2}\right)$. Consequently, to maximize $H(X)$ for a given $H\left(X_{1}\right)$, it suffices to maximize $H\left(X_{2}\right)$.

The optimization problem for $H\left(X_{i}\right)$ can be written as

$$
\max _{a_{i k}} H\left(X_{i}\right), \quad \text { s.t. } \quad \sum_{k=0}^{K_{i}} a_{i k}=1, \sum_{k=0}^{K_{i}} a_{i k} k \ell_{1}=\mathcal{E}_{i},
$$

with $K_{i} \ell_{i}=\mathcal{A}_{i}$. The solution of this optimization problem has been given in [22] as

$$
a_{i k}=\frac{t_{i}^{k}}{\sum_{j=0}^{K_{i}} t_{i}^{j}}, \quad k=0, \cdots, K_{i},
$$

where $t_{i} \in[0,1]$ is the solution of $\sum_{k=0}^{K_{i}}\left(1-\frac{k \mathcal{A}_{i}}{K_{i} \varepsilon_{i}}\right) t^{k}=0$, which exists if $\mathcal{E}_{i} \leq \frac{\mathcal{A}_{i}}{2}$ [22]. To guarantee the existence of $t_{i} \in[0,1]$, we choose $\mathcal{E}_{i}=\alpha \mathcal{A}_{i}$ which suffices since $\alpha \leq \frac{1}{2}$.

Note that the obtained distribution maximizes $H\left(X_{1}\right)$ and $H\left(X_{1}+X_{2}\right)$ as long as $k_{1} \ell_{1} \neq k_{2} \ell_{2} \forall k_{i} \in\left\{1, \cdots, K_{i}\right\}$. However, if this condition is not satisfied, then we can still use these distribution to obtain an achievable rate.

2) Achievable Rate Region: The achievable rate region corresponding to these distributions is obtained using the following steps. First $\mathcal{A}$ is split to $\mathcal{A}_{1}$ and $\mathcal{A}_{2}$. Then, we set $\mathcal{E}_{i}=\alpha \mathcal{A}_{i}, i=1,2$, and we choose $K_{1}, K_{2} \geq 1$. Given $\mathcal{A}_{i}$ and $\mathcal{E}_{i}$, we obtain the distributions on $X_{1}$ and $X_{2}$ from (22) and (24), which are then used to evaluate $R_{2}$ and $R_{1}$. This is stated formally next.

Definition 2: Let $Q$ be defined as

$$
\begin{aligned}
\mathcal{Q}=\left\{\left(\boldsymbol{q}_{1}, \boldsymbol{q}_{2}\right) \mid \boldsymbol{q}_{i}=\left(\mathcal{A}_{i}, K_{i}\right) \in \mathbb{R}_{+} \times \mathbb{N}_{+},\right. & i \in\{1,2\}, \\
& \left.\mathcal{A}_{1}+\mathcal{A}_{2}=\mathcal{A}\right\} .
\end{aligned}
$$

Further, denote by $p_{X_{i}}^{*}$ the distribution of $X_{i}$ satisfying $\mathbb{E}\left[X_{i}\right]=\mathcal{E}_{i}=\alpha \mathcal{A}_{i}$ obtained from (22) and (24), $i \in\{1,2\}$.

Theorem 3: The region $\mathcal{R}_{D}\left(\boldsymbol{q}_{1}, \boldsymbol{q}_{2}\right)$ described by rate pairs $\left(R_{1}, R_{2}\right) \in \mathbb{R}_{+}^{2}$ satisfying

$$
\begin{aligned}
& R_{2} \leq I\left(X_{2} ; Y_{2}\right), \\
& R_{1} \leq I\left(X_{1} ; Y_{1} \mid X_{2}\right)
\end{aligned}
$$

is achievable, where $\left(\boldsymbol{q}_{1}, \boldsymbol{q}_{2}\right) \in \mathcal{Q}, \boldsymbol{q}_{i}=\left(\mathcal{A}_{i}, K_{i}\right) i=1,2$, and $X_{i}$ is distributed according to $p_{X_{i}}^{*}$. Thus, the capacity region $\mathcal{C}$ of the 2-user $\mathrm{OBC}$ satisfies $\mathcal{C} \supseteq \underline{\mathcal{e}}_{D}=$ $\bigcup_{\left(\boldsymbol{q}_{1}, \boldsymbol{q}_{2}\right) \in \mathcal{Q}} \mathcal{R}_{D}\left(\boldsymbol{q}_{1}, \boldsymbol{q}_{2}\right)$.

Proof: The achievability of $\mathcal{R}_{D}\left(\boldsymbol{q}_{1}, \boldsymbol{q}_{2}\right)$ follows by SC [24], [26] and the inner bound follows by taking the union over the set of feasible parameters $Q$.

Next, we focus on the capacity region at high SNR.

\section{HIGH SNR ANALYSIS}

The channel has high SNR if $\frac{\varepsilon}{\sigma_{2}} \gg 1$. In this case, the upper bound $\bar{C}_{\alpha}^{[h]}(\mathcal{A}, \sigma)=\frac{1}{2} \log \left(1+\frac{c \mathcal{A}^{2}}{\sigma^{2}}\right)$ given in (8) will be useful, where $c=\min \left\{\frac{1}{2 \pi e}, \frac{e \alpha^{2}}{2 \pi}\right\}$. Using this bound, the outer bound of Theorem 1 becomes as given in the following corollary.

Corollary 1: Let $\overline{\mathcal{R}}^{[h]}(\rho)$ be the set of rate pairs $\left(R_{1}, R_{2}\right) \in$ $\mathbb{R}_{+}^{2}$ satisfying

$$
\begin{aligned}
R_{1} & \leq \frac{1}{2} \log \left(1+\frac{c \rho^{2} \mathcal{A}^{2}}{\sigma_{1}^{2}}\right) \\
R_{2} & \leq \frac{1}{2} \log \left(1+\frac{c\left(1-\rho^{2}\right) \mathcal{A}^{2}}{\sigma_{2}^{2}+c \rho^{2} \mathcal{A}^{2}}\right),
\end{aligned}
$$

where $\rho \in[0,1]$ and $c$ is given in (9). Then $\mathcal{C} \subseteq \overline{\mathcal{C}}^{[h]}=$ $\bigcup_{\rho \in[0,1]} \overline{\mathcal{R}}^{[h]}(\rho)$ at high SNR.

Proof: Follows from Theorem 1 and the upper bound (8).

This outer bound is easily computable. Fig. 3 shows this bound for $\alpha=1 / 2$ and $\alpha=1 / 5$, along with the TG inner bounds $\underline{\mathfrak{e}}_{T}$ and $\underline{\mathcal{C}}_{T}^{\prime}$ (Theorem 2 and Proposition 1), and the convexified inner bounds $\mathrm{CH}\left(\underline{\mathfrak{e}}_{T}\right)$ and $\mathrm{CH}\left(\underline{\mathfrak{e}}_{T}^{\prime}\right)$. Convexification is achieved by time-sharing. The achievable rate due Theorem 3 is not shown since its performance at high SNR is comparable to $\mathrm{CH}\left(\underline{\mathfrak{e}}_{T}\right)$. This figure shows that $\mathrm{CH}\left(\underline{\mathfrak{e}}_{T}\right)$ is fairly tight. The simpler inner bound $\mathrm{CH}\left(\underline{\mathfrak{C}}_{T}^{\prime}\right)$ is not as tight, but it is useful for bounding the capacity within a constant gap as we show next. 


\section{A. High SNR Symmetric-Capacity within a Constant}

The symmetric-capacity is defined as $\max _{(R, R) \in \mathcal{C}} R$. We denote this capacity at high SNR by $C_{h}$. An upper bound on $C_{h}$ can be found by enlarging the feasible set of this maximization from $\mathcal{C}$ to an outer bound on $\mathcal{C}$. Thus, $C_{h} \leq$ $\bar{C}_{h}=\max _{(R, R) \in \overline{\mathrm{e}}^{[h]}} R$. Solving this problem is equivalent to finding the point on the boundary of $\overline{\mathcal{C}}^{[h]}$ that lies on the line $R_{2}=R_{1}$.

Thus, we first have to characterize the boundary of $\overline{\mathcal{C}}^{[h]}$. The region $\overline{\mathcal{C}}^{[h]}$ is defined by the union of rectangles given by $\left(R_{1}, R_{2}\right) \in \mathbb{R}_{+}^{2}$ that satisfy (27)-(28). The boundary of $\overline{\mathcal{C}}^{[h]}$ clearly consists of the union of the outermost corner points of such rectangles. Thus, this boundary is characterized by the following parametric curve

$$
\begin{aligned}
& R_{1}=\frac{1}{2} \log \left(1+\frac{c \rho^{2} \mathcal{A}^{2}}{\sigma_{1}^{2}}\right) \\
& R_{2}=\frac{1}{2} \log \left(1+\frac{c\left(1-\rho^{2}\right) \mathcal{A}^{2}}{\sigma_{2}^{2}+c \rho^{2} \mathcal{A}^{2}}\right),
\end{aligned}
$$

with $\rho \in[0,1]$. Now, we can solve $\bar{C}_{h}=\max _{(R, R) \in \overline{\mathrm{e}}^{[h]}} R$ by

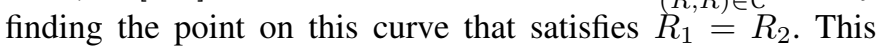
point corresponds to

$$
\rho^{2}=-\left(\frac{\sigma_{2}^{2}+\sigma_{1}^{2}}{2 c \mathcal{A}^{2}}\right)+\sqrt{\left(\frac{\sigma_{2}^{2}+\sigma_{1}^{2}}{2 c \mathcal{A}^{2}}\right)^{2}+\frac{\sigma_{1}^{2}}{c \mathcal{A}^{2}}} .
$$

Note that $\rho^{2}>0$ and $\rho^{2}<\frac{\sigma_{1}}{\sqrt{c} \mathcal{A}}$ which is smaller than 1 at high SNR. Substituting in (29) yields

$$
R_{1} \leq \frac{1}{2} \log \left(\frac{1}{2}+\frac{1}{2} \sqrt{\left(1+\frac{\sigma_{2}^{2}}{\sigma_{1}^{2}}\right)^{2}+\frac{4 c \mathcal{A}^{2}}{\sigma_{1}^{2}}}\right) .
$$

Since we are concerned with the high-SNR symmetric rate, we can neglect $\left(1+\frac{\sigma_{2}^{2}}{\sigma_{1}^{2}}\right)^{2}$ in comparison with $\frac{4 c \mathcal{A}^{2}}{\sigma_{1}^{2}}$ to obtain the symmetric capacity upper bound

$$
C_{h} \leq \bar{C}_{h}=\frac{1}{2} \log \left(\frac{1}{2}+\frac{\sqrt{c} \mathcal{A}}{\sigma_{1}}\right) .
$$

A lower bound on $C_{h}$ can be obtained from the achievable rate region in Proposition 1. We will neglect $\sigma_{2}^{2}$ in the constraint on $R_{2}$ at high SNR to obtain the rate constraint $R_{2} \leq \frac{1}{2} \log \left(1+\frac{(1-\beta)^{2}}{\beta^{2}}\right)$. Similar to above, to obtain an achievable symmetric-rate, we equate the rate constraint on $R_{1}$ from Proposition 1 and this constraint on $R_{2}$, and solve for $\beta$. This yields

$$
\beta^{*}=\sqrt{\frac{2 \sigma_{1}}{\alpha \mathcal{A}}},
$$

which is in $[0,1]$ for high SNR, and which when substituted in one of the constraints leads to an achievable symmetric-rate given by

$$
\underline{C}_{h}=\frac{1}{2} \log \left(1+\frac{\alpha \mathcal{A}}{3 \sigma_{1}}\right) .
$$

This leads to the following corollary.

Corollary 2: The high-SNR symmetric-capacity $C_{h}$ of the 2-user OBC satisfies $\underline{C}_{h} \leq C_{h} \leq \bar{C}_{h}$ where $\underline{C}_{h}$ and $\bar{C}_{h}$

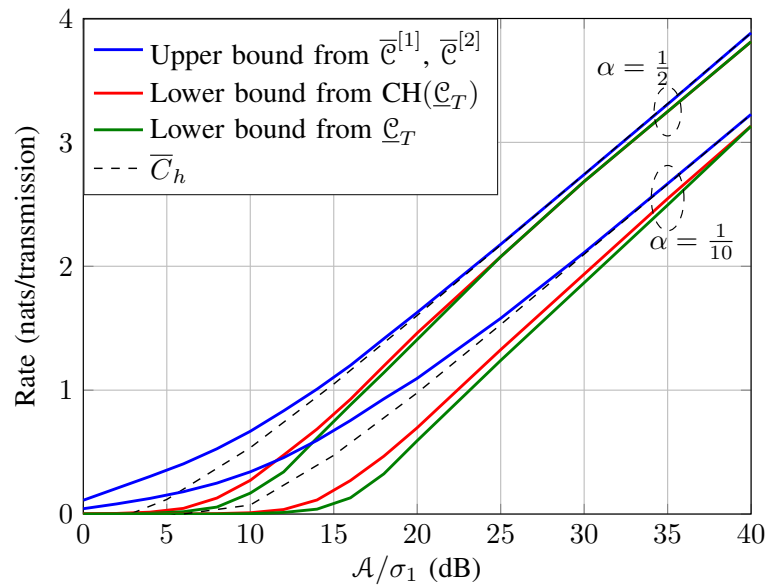

Fig. 4: Symmetric capacity bounds versus SNR for an OBC with $\sigma_{2}=2 \sigma_{1}$.

are given in (31) and (33). Furthermore, the gap between the two bounds satisfies $\bar{C}_{h}-\underline{C}_{h} \stackrel{\frac{\varepsilon}{\sigma_{2}} \rightarrow \infty}{\longrightarrow} \frac{1}{2} \log \left(\frac{3 \sqrt{c}}{\alpha}\right) \leq 0.34$ nats/transmission.

Proof: The bounds follow from the above discussion. By approximating the bounds at high SNR by $\frac{1}{2} \log \left(\frac{\alpha \mathcal{A}}{3 \sigma_{1}}\right)$ and $\frac{1}{2} \log \left(\frac{\sqrt{c} \mathcal{A}}{\sigma_{1}}\right)$, we get the gap $\frac{1}{2} \log \left(\frac{3 \sqrt{c}}{\alpha}\right)$. For $\alpha<\frac{1}{e}, c=$ $\frac{e \alpha^{2}}{2 \pi}$ and this gap evaluates to 0.34 nats. Otherwise, $c=\frac{1}{2 \pi e}$ and the gap is $\leq 0.34$ nats.

We recall that the achievability of $\underline{C}_{h}$ is simple. The transmitter simply splits $\mathcal{A}$ into $\mathcal{A}_{1}=\beta^{*} \mathcal{A}$ and $\mathcal{A}_{1}=\left(1-\beta^{*}\right) \mathcal{A}$ where $\beta^{*}$ is given in (32), and sends $X=X_{1}+X_{2}$ where $X_{i}$ follows a TG distribution over $\left[0, \mathcal{A}_{i}\right]$ with the parameters given in Sec. IV-A2. To see at which SNR the statement of Corollary 2 starts to hold, we plot symmetric rate upper and lower bounds in Fig. 4. The asymptotic upper bound $\bar{C}_{h}$ is also plotted. The upper and lower bounds are obtained from the regions $\overline{\mathrm{C}}^{[i]}, i \in\{1,2\}$, and $\underline{\mathcal{E}}_{T}$, respectively, by finding the intersection of the boundary of those regions with the line $R_{1}=R_{2}$. Those bounds are found numerically, where the tighter between $\bar{\complement}^{[1]}$ and $\bar{\complement}^{[2]}$ is selected. This figure shows that bounds become parallel (constant gap) around $\frac{\mathcal{A}}{\sigma_{1}}=25 \mathrm{~dB}$ for $\alpha=1 / 2$, and around $\frac{\mathcal{A}}{\sigma_{1}}=35 \mathrm{~dB}$ for $\alpha=1 / 10$. This threshold is higher for smaller $\alpha$ because the statement of Corollary 2 requires $\alpha \mathcal{A} / \sigma_{1}$ to be large (see (31) and (33)).

\section{B. Symmetric-Capacity versus TDMA}

It is interesting to compare the achievable symmetric-rate using SC with that of simple time-division multiple access (TDMA), both under TG distributions. In TDMA, the transmitter decomposes the OBC into two P2P channels by sending to user 1 in a fraction $\tau \in[0,1]$ of time, and to user 2 in the remaining time. Over each $\mathrm{P} 2 \mathrm{P}$ channel, the transmitter uses a $(\mathcal{A}, \mu, \nu)$-TG distribution with mean $\tilde{\mu}=\mathcal{E}$ (16). Denote the highest achievable rate of users $i$ using this scheme by $\bar{R}_{i}$. According to Theorem 2, this rate can be written as $\bar{R}_{i}=\max _{\mu, \nu} \frac{1}{2} \log \left(\frac{\nu^{2}}{\tilde{\nu}^{2}}+\frac{\nu^{2}}{\sigma_{i}^{2}}\right)-\phi$, where $\phi$ is defined in 


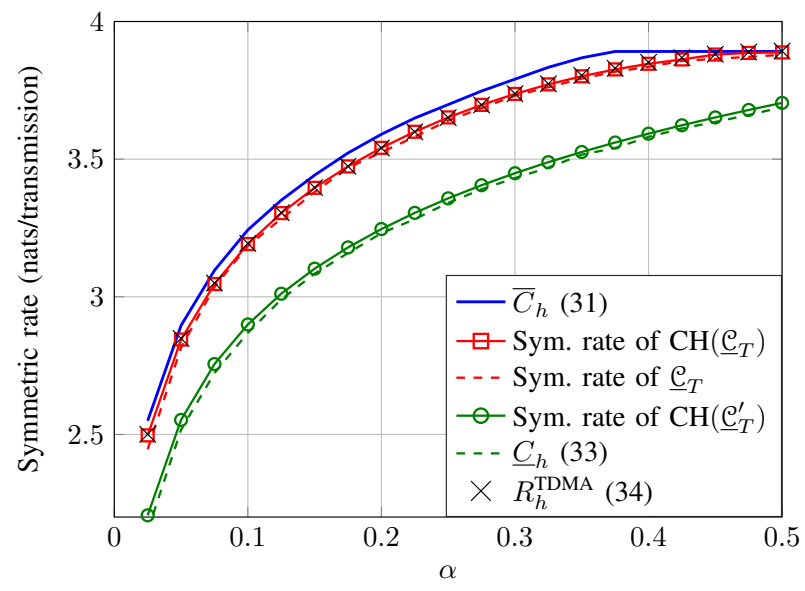

(a) $\sigma_{2}=\sigma_{1}$.

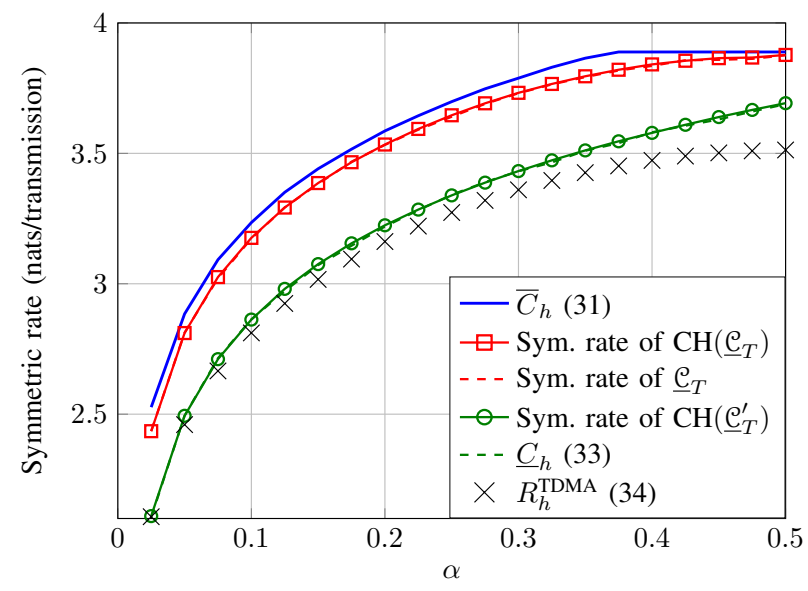

(b) $\sigma_{2}=4 \sigma_{1}$.

Fig. 5: Achievable symmetric-rates as a function of $\alpha$ for an $\mathrm{OBC}$ with $\frac{\mathcal{A}}{\sigma_{1}}=40 \mathrm{~dB}$. The upper bound (31) is also shown for comparison.

Theorem 2, $\tilde{\nu}$ in (17), and where the maximization is over all $\mu$ and $\nu$ such that $\tilde{\mu}=\mathcal{E}$. Thus, for a given $\tau \in[0,1]$, TDMA achieves $R_{1} \leq \tau \bar{R}_{1}$ and $R_{2} \leq(1-\tau) \bar{R}_{2}$. The maximum achievable symmetric-rate is thus

$$
R_{h}^{\mathrm{TDMA}}=\frac{\bar{R}_{1} \bar{R}_{2}}{\bar{R}_{1}+\bar{R}_{2}}
$$

Let us study $R_{h}^{\mathrm{TDMA}}$ at high SNR. Since the TG distribution approaches the high SNR capacity of the P2P IM-DD channel [10] which is closely captured by $\log \left(\sqrt{c} \mathcal{A} / \sigma_{i}\right),{ }^{2}$ then we have $\bar{R}_{i} \stackrel{\frac{\alpha \mathcal{A}}{\sigma_{i}} \rightarrow \infty}{\longrightarrow} \log \left(\sqrt{c} \mathcal{A} / \sigma_{i}\right)$. Thus, by writing $\sigma_{2}=k \sigma_{1}$ with $k \geq 1$, we have

$$
\begin{gathered}
R_{h}^{\mathrm{TDMA}} \stackrel{\frac{\alpha \mathcal{A}}{\sigma_{2}} \rightarrow \infty}{\longrightarrow} \log \left(\frac{\sqrt{c} \mathcal{A}}{\sigma_{1}}\right) \frac{\log \left(\frac{\sqrt{c} \mathcal{A}}{\sigma_{1}}\right)-\log (k)}{\log \left(\frac{c \mathcal{A}^{2}}{\sigma_{1}^{2}}\right)-\log (k)} \\
\stackrel{\frac{\alpha \mathcal{A}}{\sigma_{2}} \rightarrow \infty}{\longrightarrow} \log \left(\frac{\sqrt{c} \mathcal{A}}{\sigma_{1}}\right)\left(\frac{1}{2}-\frac{\log (\sqrt{k})}{\log \left(\frac{c \mathcal{A}^{2}}{\sigma_{1}^{2}}\right)}\right) \\
=\frac{1}{2} \log \left(\frac{\sqrt{c} \mathcal{A}}{\sigma_{1}}\right)-\frac{1}{2} \log (\sqrt{k}) .
\end{gathered}
$$

Now we compare $R_{h}^{\mathrm{TDMA}}$ with the high-SNR symmetric capacity $C_{h}$. By Corollary 2 we have

$$
\begin{aligned}
R_{h}^{\mathrm{TDMA}}-C_{h} & \leq R_{h}^{\mathrm{TDMA}}-\underline{C}_{h} \\
\stackrel{\frac{\alpha \mathcal{A}}{\sigma_{2}} \rightarrow \infty}{\longrightarrow} & \frac{1}{2} \log \left(\frac{\sqrt{c} \mathcal{A}}{\sigma_{1}}\right)-\frac{1}{2} \log (\sqrt{k})-\frac{1}{2} \log \left(\frac{\alpha \mathcal{A}}{3 \sigma_{1}}\right) \\
& =\frac{1}{2} \log \left(\frac{3 \sqrt{c}}{\alpha}\right)-\frac{1}{2} \log (\sqrt{k}) .
\end{aligned}
$$

This quantity becomes negative if $k$ is large. In this case, which occurs if user 1 is much close to the transmitter than user 2 e.g., TDMA is suboptimal at high SNR. On the other hand, if $k=1$, then $\bar{R}_{1}=\bar{R}_{2}$, and $R_{h}^{\mathrm{TDMA}}=\frac{\bar{R}_{1}}{2} \stackrel{\frac{\alpha \mathcal{A}}{\sigma_{2}} \rightarrow \infty}{\longrightarrow}$

\footnotetext{
${ }^{2}$ The gap between $\log \left(\sqrt{c} \mathcal{A} / \sigma_{i}\right)$ and capacity is $<0.1$ nats [10], and will be ignored here.
}

$\frac{1}{2} \log \left(\frac{\sqrt{c} \mathcal{A}}{\sigma_{1}}\right)$ which coincides with the upper bound given in Corollary 2. Thus, TDMA is optimal at high SNR if $\sigma_{1}=\sigma_{2}$.

Since $R_{h}^{\text {TDMA }}$ is achieved by time-sharing between achievable rate pairs in $\underline{\mathcal{C}}_{T}$, then the achievable symmetric-rate in $\mathrm{CH}\left(\underline{\mathfrak{E}}_{T}\right)$ is generally higher than $R_{h}^{\text {TDMA }}$. Fig. 5 shows the achievable symmetric-rate versus $\alpha$ at high SNR. From this figure, we can see that when $\sigma_{1}=\sigma_{2}$, TDMA becomes nearly optimal confirming the above statement. When $\sigma_{2}>\sigma_{1}$, SC outperforms TDMA. We can also note that the achievable symmetric-rates due to $\underline{\mathcal{E}}_{T}$ and $\underline{\mathcal{C}}_{T}^{\prime}$ are almost equal to those due to $\mathrm{CH}\left(\underline{\mathrm{e}}_{T}\right)$ and $\mathrm{CH}\left(\underline{\mathrm{e}}_{T}^{\prime}\right)$, respectively. Consequently, timesharing is not necessary at high SNR from a symmetriccapacity perspective. The gap between the symmetric rate corresponding to $\mathrm{CH}\left(\underline{\mathrm{e}}_{T}^{\prime}\right)$ and $\bar{C}_{h}$ can be seen to be less than 0.34 nats, confirming Corollary 2.

In conclusion, TDMA is a good strategy in terms of symmetric rate at high SNR if the receivers have similar channel qualities to the transmitter (similar relative position with respect to the light source e.g.). Otherwise, SC is better, but time-sharing is not necessary at high SNR. Next, we extend this result to prove that $\underline{\mathcal{C}}_{T}^{\prime}$ and $\overline{\mathcal{C}}^{[h]}$ are within a constant gap at high SNR.

\section{High SNR Capacity Region within a Constant}

First, we describe the boundaries of $\overline{\mathcal{C}}^{[h]}$ and $\underline{\mathcal{C}}_{T}^{\prime}$ by a function $R_{2}=f\left(R_{1}\right)$. We start with the outer bound whose boundary ${ }^{3}$ is described by

$$
R_{2}=\frac{1}{2} \log \left(\frac{\sigma_{2}^{2}+c \mathcal{A}^{2}}{\sigma_{2}^{2}+\sigma_{1}^{2}\left(e^{2 R_{1}}-1\right)}\right) .
$$

On the other hand, the boundary of the inner bound $\underline{\mathfrak{C}}_{T}^{\prime}$ is described by

$$
R_{2}=\frac{1}{2} \log \left(\frac{9 \sigma_{2}^{2}+\alpha^{2} \beta^{2} \mathcal{A}^{2}+\alpha^{2}(1-\beta)^{2} \mathcal{A}^{2}}{9 \sigma_{2}^{2}+9 \sigma_{1}^{2}\left(e^{2 R_{1}}-1\right)}\right) .
$$

${ }^{3}$ We refer here and henceforth to the Pareto-boundary, which is in this case the boundary of the region excluding the axes. 
Hence, the gap between the two functions is

$$
\Delta=\frac{1}{2} \log \left(\frac{\sigma_{2}^{2}+c \mathcal{A}^{2}}{\sigma_{2}^{2}+\frac{1}{9} \alpha^{2} \mathcal{A}^{2}\left(\beta^{2}+(1-\beta)^{2}\right)}\right) .
$$

This gap is maximum at $\beta=\frac{1}{2}$. Since $\underline{\mathcal{C}}_{T}^{\prime}$ is not convex, this maximium lies in the region of non-convexity of $\underline{\mathfrak{C}}_{T}^{\prime}$ (Fig. 3). To reduce this gap, we should convexify this region.

To obtain the boundary of $\mathrm{CH}\left(\underline{\mathrm{e}}_{T}^{\prime}\right)$, one has to find $\beta$ for which the tangent to the boundary of $\underline{\mathcal{e}}_{T}^{\prime}$ passes through $\left(\bar{R}_{1}^{\prime}, 0\right)$, where $\bar{R}_{1}^{\prime}=\max _{\left(R_{1}, R_{2}\right) \in \underline{\mathfrak{e}}_{T}^{\prime}} R_{1}$. Instead of finding this $\beta$, motivated by the above observation that the symmetricrates due to $\underline{\mathcal{C}}_{T}^{\prime}$ and $\mathrm{CH}\left(\underline{\mathfrak{C}}_{T}^{\prime}\right)$ are close at high SNR, we use $\beta^{*}$ from (32). This does not necessarily convexify the region, but serves our goal.

The boundary of the inner bound obtained by combining the region $\underline{\mathrm{e}}_{T}^{\prime}$ with time-sharing between $\left(\bar{R}_{1}^{\prime}, 0\right)$ and the symmetric-rate point $\left(\underline{C}_{h}, \underline{C}_{h}\right)$ is described by

$$
R_{2}= \begin{cases}\frac{1}{2} \log \left(\frac{9 \sigma_{2}^{2}+\left(\alpha^{2} \beta^{2}+\alpha^{2}(1-\beta)^{2}\right) \mathcal{A}^{2}}{9 \sigma_{2}^{2}+9 \sigma_{1}^{2}\left(e^{2 R_{1}}-1\right)}\right), & R_{1} \leq \underline{C}_{h} \\ \frac{\underline{C}_{h}\left(R_{1}-\bar{R}_{1}^{\prime}\right)}{\underline{C}_{h}-\bar{R}_{1}^{\prime}}, & R_{1}>\underline{C}_{h},\end{cases}
$$

where $\bar{R}_{1}^{\prime}=\frac{1}{2} \log \left(1+\frac{\alpha^{2} \mathcal{A}^{2}}{9 \sigma_{1}^{2}}\right)$ (21). The first case corresponding to condition $R_{1} \leq \underline{C}_{h}$ describes the portion of the boundary for $\beta \in\left[0, \beta^{*}\right]$, and the second describes the remaining portion. It is worth to note that the slope of the second portion of this boundary is -1 at high SNR.

For $R_{1} \leq \underline{C}_{h}, \Delta$ is maximum for $\beta=\beta^{*}$ since $\Delta$ is increasing in $\beta \in\left[0, \frac{1}{2}\right]$ and $\beta^{*}<\frac{1}{2}$ for $\mathcal{A}$ large. By noting that $\beta^{*} \stackrel{\frac{\varepsilon}{\sigma_{2}} \rightarrow \infty}{\longrightarrow} 0$, we conclude that $\Delta \stackrel{\frac{\varepsilon}{\sigma_{2}} \rightarrow \infty}{\longrightarrow} \log \left(\frac{3 \sqrt{c}}{\alpha}\right)$. For $R_{1}>\underline{C}_{h}$, we bound the gap by noting that the slope of the boundary of $\overline{\mathcal{C}}^{[h]}$ described by (35) is in $>-1$. In particular, it is equal to -1 at the point of intersection of the outer bound with the $R_{1}$ axis, $\left(\bar{C}_{1}, 0\right)$, where $\bar{C}_{1}=\frac{1}{2} \log \left(1+\frac{c \mathcal{A}^{2}}{\sigma_{1}^{2}}\right)(8)$. Therefore, the region defined by the two axes and the line $R_{2}=-R_{1}+\bar{C}_{1}$ is an outer bound on the capacity region. Since the boundaries of both the inner bound (38) and this outer bound are linear with slopes -1 at high SNR, the gap can be calculated at one of the extremes $R_{1}=\underline{C}_{h}$ or $R_{1}=$ $\bar{R}_{1}^{\prime}$. This gap is given by $\log \left(\frac{3 \sqrt{c}}{\alpha}\right)$ as for the first portion. Consequently, we have the following corollary.

Corollary 3: At high SNR, the capacity region of the 2user OBC is bounded as $\underline{\mathcal{e}}^{[h]} \subset \mathcal{C} \subseteq \overline{\mathcal{C}}^{[h]}$ where $\underline{\mathfrak{e}}^{[h]}=$ $\left\{\left(R_{1}, R_{2}\right) \in \mathbb{R}_{+}^{2} \mid\left(R_{1}, R_{2}+\delta\right) \in \overline{\mathrm{C}}^{[h]}\right\}$ with $\delta=\log \left(\frac{3 \sqrt{c}}{\alpha}\right)$ and $\overline{\mathcal{C}}^{[h]}$ is defined in Corollary 1. Furthermore, $\delta \leq 0.68$ nats/transmission.

Proof: The outer bound follows from Corollary 1. The inner bound follows from the above discussion showing that the gap between the inner and outer bounds in the $R_{2}$ direction is $\log \left(\frac{3 \sqrt{c}}{\alpha}\right)$. This gap is equal to 0.68 nats for $\alpha<1 / e$ and less than 0.68 nats otherwise.

Using this corollary, the boundary of $\mathcal{C}$ at high SNR lies between $f\left(R_{1}\right)-0.68$ nats and $f\left(R_{1}\right)$ where $f\left(R_{1}\right)$ is the function defined by the right-hand side of (35). It is important to note here that the inner bound $\underline{\mathcal{C}}_{T}$ (Theorem 2) combined with time-sharing, i.e., $\mathrm{CH}\left(\underline{\mathfrak{e}}_{T}\right)$, is closer to the outer bound

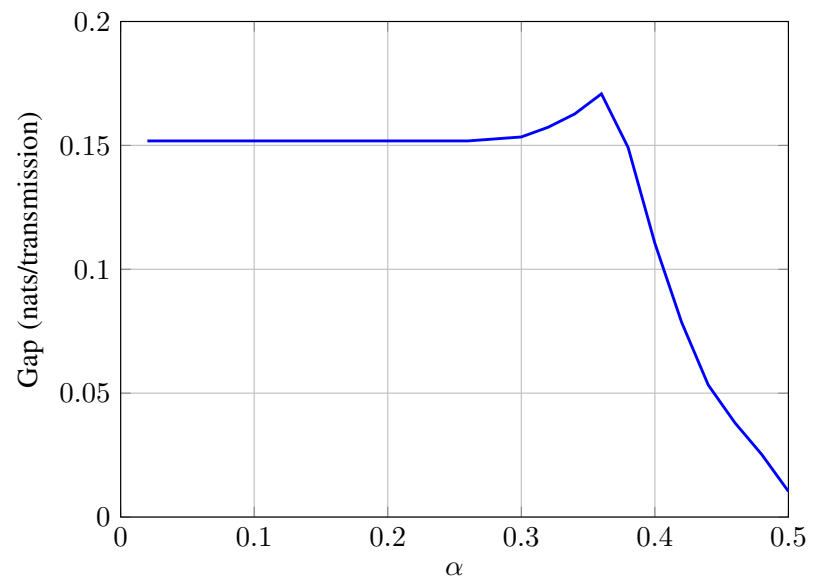

Fig. 6: The maximum gap between the outer bound $\overline{\mathcal{C}}^{[h]}$ and the inner bound $\mathrm{CH}\left(\underline{\mathrm{e}}_{T}\right)$ in the $R_{2}$ direction for an $\mathrm{OBC}$ with $\frac{\varepsilon}{\sigma_{2}}=40 \mathrm{~dB}$ and $\sigma_{2}=2 \sigma_{1}$.

than $\mathrm{CH}\left(\underline{\mathrm{e}}_{T}^{\prime}\right)$, and hence the gap is smaller than 0.68 nats. Fig. 6 shows the maximum over $R_{1}$ of the gap in the $R_{2}$ direction between $\bar{\complement}^{[h]}$ and $\underline{\mathcal{E}}_{T}$ at high SNR.

\section{LOW AND MOdERATE SNR}

This section focuses on the OBC with low/moderate SNR. We characterize the channel capacity and low SNR first, and discuss its capacity at moderate SNR afterwards.

\section{A. Low SNR}

The tightest upper bound on the IM-DD P2P channel capacity at low SNR is given by $\bar{C}_{\alpha}^{[3]}(\mathcal{A}, \sigma)$ in Lemma 1 , since it coincides with the low-SNR capacity [9]. We shall see that $\bar{C}_{\alpha}^{[3]}(\mathcal{A}, \sigma)$ leads to an outer bound which is tight at low SNR.

At low SNR, the inner bound $\underline{\mathcal{C}}_{D}$ becomes better than $\underline{\mathcal{e}}_{T}$. The reason is that discrete input distributions generally outperform continuous ones in IM-DD channels [20], [22], especially at moderate/low SNR. Unfortunately, $\underline{\mathcal{E}}_{D}$ does not have a closed-form expression. However, we know that the discrete distribution in Sec. IV-B achieves the low-SNR IMDD P2P channel capacity [9]. In particular, at low SNR, on-off keying $(\mathrm{OOK})$ is optimal leading to the following statement.

Theorem 4: At low SNR, the capacity region $\mathcal{C}$ of the 2-user $\mathrm{OBC}$ is achieved by OOK and TDMA, and it asymptotically coincides with

$$
\widehat{\mathrm{C}}^{[3]}=\left\{\left(R_{1}, R_{2}\right) \in \mathbb{R}_{+}^{2} \mid \sigma_{1}^{2} R_{1}+\sigma_{2}^{2} R_{2} \leq \frac{\alpha(1-\alpha) \mathcal{A}^{2}}{2}\right\} .
$$

Proof: We start with the outer bound $\overline{\mathcal{C}}^{[3]}=$ $\bigcup_{\rho \in[0,1]} \overline{\mathcal{R}}^{[3]}(\rho)$ given in Theorem 1, where $\overline{\mathcal{R}}^{[3]}(\rho)$ is the set of rate pairs $\left(R_{1}, R_{2}\right) \in \mathbb{R}_{+}^{2}$ that satisfy

$$
\begin{aligned}
& R_{1} \leq \frac{1}{2} \log \left(1+\frac{\rho^{2} \alpha(1-\alpha) \mathcal{A}^{2}}{\sigma_{1}^{2}}\right), \\
& R_{2} \leq \frac{1}{2} \log \left(1+\frac{\left(1-\rho^{2}\right) \alpha(1-\alpha) \mathcal{A}^{2}}{\sigma_{2}^{2}+\rho^{2} \alpha(1-\alpha) \mathcal{A}^{2}}\right) .
\end{aligned}
$$




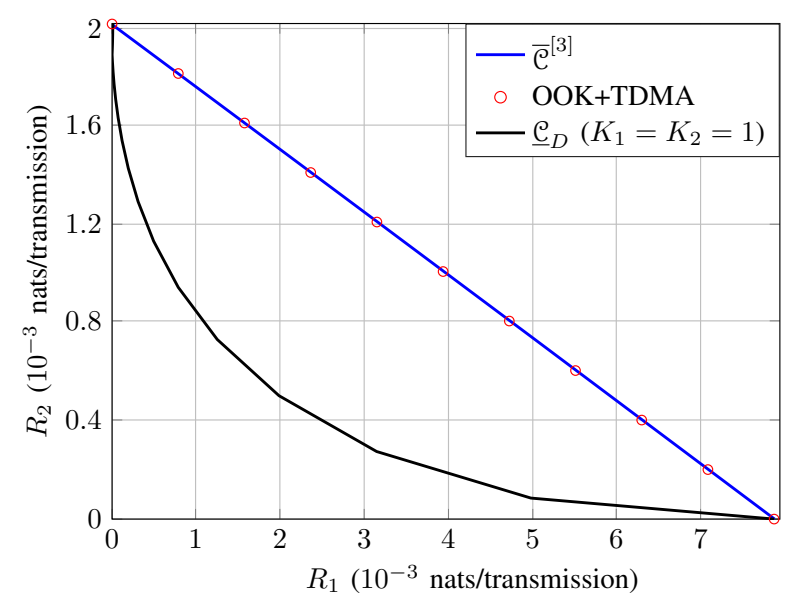

(a) Low SNR capacity of an OBC with $\alpha=0.2, \sigma_{2}=2 \sigma_{1}$, and $\frac{\mathcal{A}}{\sigma_{1}}=-5 \mathrm{~dB}$.

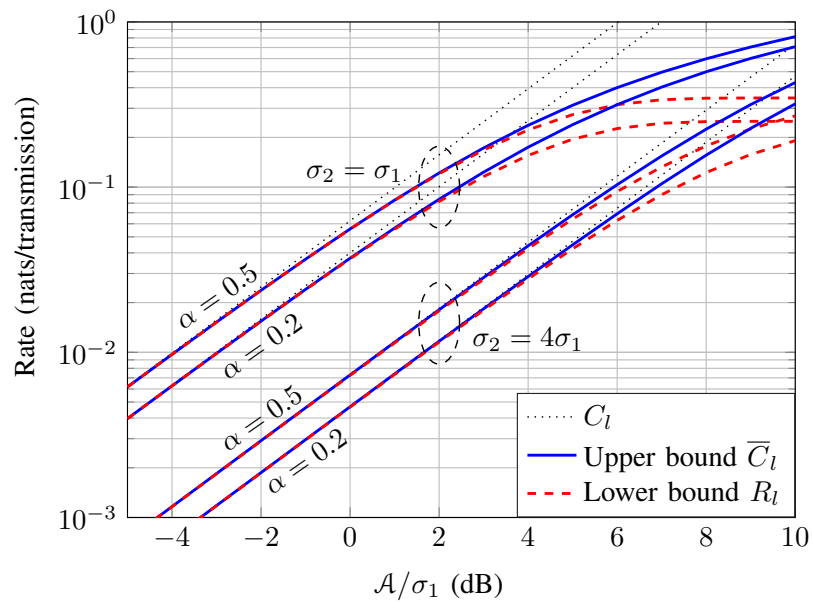

(b) Symmetric capacity bounds versus $\frac{\mathcal{A}}{\sigma_{1}}$.

Fig. 7: Low SNR capacity region and symmetric capacity bounds.

Then, we relax those constraints to $R_{1} \leq \frac{\rho^{2} \alpha(1-\alpha) \mathcal{A}^{2}}{2 \sigma_{1}^{2}}$ and $R_{2} \leq \frac{\left(1-\rho^{2}\right) \alpha(1-\alpha) \mathcal{A}^{2}}{2 \sigma_{2}^{2}}$ using $\log (1+x) \leq x$ and $\frac{a}{b+c} \leq \frac{a}{b}$ for positive $a, b$, and $c$. This leads to an outer bound given by $\widehat{\mathcal{C}}^{[3]}$ in the statement of the Theorem. The region $\widehat{\mathcal{C}}^{[3]}$ is triangular shaped, with boundary described by the line segment joining the points $P_{1}=\left(\frac{\alpha(1-\alpha) \mathcal{A}^{2}}{2 \sigma_{1}^{2}}, 0\right)$ and $P_{2}=\left(0, \frac{\alpha(1-\alpha) \mathcal{A}^{2}}{2 \sigma_{2}^{2}}\right)$. Now consider OOK combined with TDMA. Denote the rate achieved by using OOK to send information to user $i$ only by $R_{\alpha}\left(\mathcal{A}, \sigma_{i}\right)$. Clearly, the points $P_{1}^{\prime}=\left(R_{\alpha}\left(\mathcal{A}, \sigma_{1}\right), 0\right)$ and $P_{2}^{\prime}=\left(0, R_{\alpha}\left(\mathcal{A}, \sigma_{2}\right)\right)$ are achievable. Time sharing between those points achieves any rate pair on the line segment between them. This defines a triangular shaped inner bound $\widehat{\mathcal{R}}$.

At this point, we have $\widehat{\mathcal{R}} \subseteq \mathcal{C} \subseteq \overline{\mathcal{C}}^{[3]} \subseteq \widehat{\mathcal{C}}^{[3]}$. Hence, $\mathcal{C}$ and $\widehat{\mathcal{C}}^{[3]}$ asymptotically coincide at low SNR if $\widehat{\mathcal{C}}^{[3]}$ and $\widehat{\mathcal{R}}$ asymptotically coincide. Since those latter have triangular shapes, it suffices to show that their corners coincide. Both $\widehat{\mathcal{C}}^{[3]}$ and $\widehat{\mathcal{R}}$ have a corner at $(0,0)$. Further, from [9], we know that $R_{\alpha}\left(\mathcal{A}, \sigma_{i}\right) \stackrel{\frac{\mathcal{A}}{\sigma_{i}} \rightarrow 0}{\longrightarrow} \frac{\alpha(1-\alpha) \mathcal{A}^{2}}{2 \sigma_{i}^{2}}$. Thus, $P_{i}$ and $P_{i}^{\prime}$ converge as SNR decreases. Therefore, $\widehat{\mathcal{C}}^{[3]}$ and $\widehat{\mathcal{R}}$ converge to the same region, which completes the proof.

As a conclusion, the simple combination of (coded) OOK and TDMA suffices for achieving the capacity region at low SNR. Fig. 7a shows the capacity region of the OBC at low SNR, for a setting with $\sigma_{2}=2 \sigma_{1}$. In this figure, $\underline{\mathcal{e}}_{D}$ is plotted with $K_{1}=K_{2}=1$. Keep in mind that while in this case $X_{1}$ and $X_{2}$ are binary, $X$ is not binary but quaternary. However, the points of maximum $R_{1}$ and $R_{2}$ (intersection with the axes) correspond to $\mathcal{A}_{2}=0$ and $\mathcal{A}_{1}=0$, respectively, and the channel input $X$ is binary at these points. Thus, the extremes of $\underline{\mathcal{E}}_{D}$ are achievable by OOK. The figure shows that timesharing between these extremes is optimal.

Based on Theorem 4, we have the following corollary.

Corollary 4: The symmetric-capacity of the OBC satisfies $C_{l} \stackrel{\frac{\mathcal{A}}{\sigma_{1}} \rightarrow 0}{\longrightarrow} \frac{\alpha(1-\alpha) \mathcal{A}^{2}}{2\left(\sigma_{1}^{2}+\sigma_{2}^{2}\right)}$.
Proof: By finding the point $(R, R)$ which lies on the boundary of $\widehat{\mathcal{C}}^{[3]}$ in Theorem 4 .

Alternatively, this corollary can be proved by finding a symmetric capacity upper bound based on $\overline{\mathcal{C}}^{[3]}$, and then comparing with the achievable symmetric rate of OOK with TDMA. An upper bound based on $\overline{\mathrm{e}}^{[3]}$ is obtained by finding $\tilde{\rho} \in[0,1]$ which makes the right-hand-sides of (39) and (40) equal. Substituting this $\tilde{\rho}$ in (39) yields the symmetric capacity upper bound $C_{l} \leq \bar{C}_{l}=\frac{1}{2} \log \left(1+\tilde{\rho}^{2} \alpha(1-\alpha) \mathcal{A}^{2} / \sigma_{1}^{2}\right)$. On the other hand, OOK combined with TDMA achieves rate pairs $\left(R_{1}, R_{2}\right) \in \mathbb{R}_{+}^{2}$ which satisfy $R_{1} \leq \tau I\left(X ; Y_{1}\right)$ and $R_{2} \leq(1-\tau) I\left(X ; Y_{2}\right)$, where $\tau \in[0,1]$ and $X \in\{0, \mathcal{A}\}$ is distributed according to $p_{X}(0)=1-\alpha$ and $p_{X}(\mathcal{A})=\alpha$. A symmetric capacity lower bound can thus be obtained as $C_{l} \geq R_{l}=\tilde{\tau} I\left(X ; Y_{1}\right)$ where $\tilde{\tau}$ is the solution of $\tau I\left(X ; Y_{1}\right)=$ $(1-\tau) I\left(X ; Y_{2}\right)$. Now we focus on low SNR. It is easy to show that $\tilde{\rho}^{2}=\frac{\sigma_{1}^{2}}{\sigma_{1}^{2}+\sigma_{2}^{2}}$ at low SNR. Thus, $\bar{C}_{l} \stackrel{\frac{\mathcal{A}}{\sigma_{1}} \rightarrow 0}{\longrightarrow} \frac{\alpha(1-\alpha) \mathcal{A}^{2}}{2\left(\sigma_{1}^{2}+\sigma_{2}^{2}\right)}$. On the other hand, $I\left(X ; Y_{i}\right) \stackrel{\frac{\mathcal{A}}{\sigma_{1}} \rightarrow 0}{\longrightarrow} \frac{\operatorname{Var}(X)}{2 \sigma_{i}^{2}}=\frac{\alpha(1-\alpha) \mathcal{A}^{2}}{2 \sigma_{i}^{2}}$ [9], where $\operatorname{Var}(X)$ is the variance of $X$. This leads to $\tilde{\tau}=\tilde{\rho}^{2}$ and $R_{l} \stackrel{\frac{\mathcal{A}}{\sigma_{1}} \rightarrow 0}{\longrightarrow} \frac{\alpha(1-\alpha) \mathcal{A}^{2}}{2\left(\sigma_{1}^{2}+\sigma_{2}^{2}\right)}$ leading to the statement of Corollary 4 . Fig. 7b shows the bounds $\bar{C}_{l}$ and $R_{l}$ versus SNR for a channel with different values of $\sigma_{2}$ and $\alpha$, where the convergence of the bounds at low SNR is obvious.

\section{B. Moderate SNR}

We have seen that TG inputs achieve the capacity region within a small gap at high SNR. We have also seen that OOK combined with TDMA is optimal at low SNR. Next, we investigate the moderate SNR regime, where SNR can neither be described as high nor low. At moderate SNR, the discrete input distribution discussed in Sec. IV-B performs fairly good, and outperforms TG.

Fig. 8 shows achievable rate regions and outer bounds for a channel with $\frac{\varepsilon}{\sigma_{2}}=5 \mathrm{~dB}$. It can be seen that $\underline{\mathcal{C}}_{D}$ is 


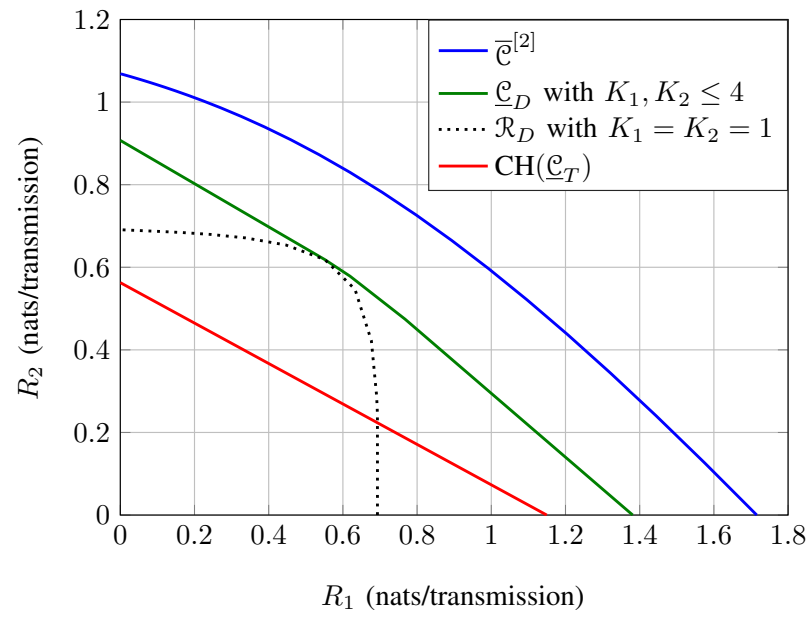

(a) $\alpha=\frac{1}{2}$.

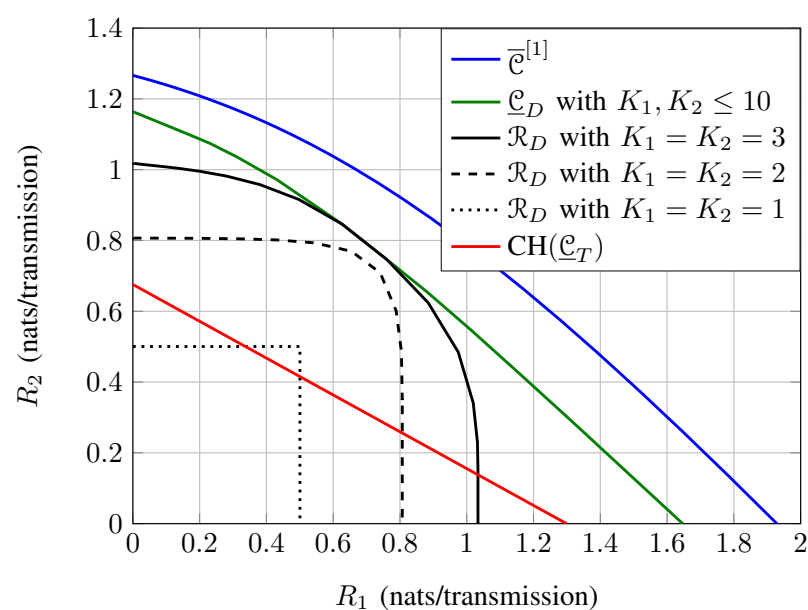

(b) $\alpha=\frac{1}{5}$.

Fig. 8: Capacity region outer and inner bounds for an $\mathrm{OBC}$ with $\frac{\varepsilon}{\sigma_{2}}=5 \mathrm{~dB}$ and $\sigma_{2}=2 \sigma_{1}$.

fairly close to the outer bound. We have restricted each of $K_{1}$ and $K_{2}$ to $\{1, \cdots, 4\}$ in Fig. $8 \mathrm{a}$ and to $\{1, \cdots, 10\}$ in Fig. 8 b. We have also plotted the achievable rate region for some $K_{1}$ and $K_{2}$. This shows that even with small $K_{1}$ and $K_{2}$, a discrete input outperforms TG at moderate SNR. This observation has several practical implications. First, a discrete input distribution which is practically simpler to realize than a continuous distribution achieves good performance. Second, an acceptable symmetric-rate is achieved at moderate SNR with a low number of mass points $\left(K_{1}=K_{2}=1\right.$ in Fig. 8a and $K_{1}=K_{2} \leq 3$ in Fig. 8 b).

\section{VII. $N$-USER OBC}

In this section, we extend the above results to the $N$-user case. We start with the outer bound.

Theorem 5: The capacity region $\mathcal{C}$ of the $N$-user $\mathrm{OBC}$ is outer bounded by $\overline{\mathcal{C}}^{[j]}=\bigcup_{\rho \in \mathcal{S}_{\rho}} \overline{\mathcal{R}}^{[j]}(\boldsymbol{\rho})$ where $j \in\{1,2,3\}$, $\overline{\mathcal{R}}^{[j]}(\boldsymbol{\rho})$ is the set of $\left(R_{1}, \cdots, R_{N}\right) \in \mathbb{R}_{+}^{N}$ that satisfy

$$
R_{i} \leq \frac{1}{2} \log \left(\frac{\sigma_{i}^{2}+\sigma_{N}^{2}\left(e^{2 \bar{C}_{\alpha}^{[j]}\left(\rho_{i+1} \mathcal{A}, \sigma_{N}\right)}-1\right)}{\sigma_{i}^{2}+\sigma_{N}^{2}\left(e^{2 \bar{C}_{\alpha}^{[j]}\left(\rho_{i} \mathcal{A}, \sigma_{N}\right)}-1\right)}\right),
$$

$i \in \mathcal{N}, \boldsymbol{\rho}=\left(\rho_{1}, \cdots, \rho_{N}\right)$, and $\mathcal{S}_{\boldsymbol{\rho}}=\left\{\boldsymbol{\rho} \in[0,1]^{N} \mid \rho_{i} \leq\right.$ $\left.\rho_{i+1} \forall i \in \mathcal{N}, \rho_{1}=0, \rho_{N+1}=1\right\}$.

Proof: The proof is similar to that of Theorem 1. Details are given in Appendix B.

The achievable rate region by the TG distribution is stated next for the $N$-user case, followed by the simplified version of this region.

Theorem 6: For $\left(\boldsymbol{p}_{1}, \cdots, \boldsymbol{p}_{N}\right) \in \mathcal{P}$ defined as

$$
\begin{array}{r}
\mathcal{P}=\left\{\left(\boldsymbol{p}_{1}, \cdots, \boldsymbol{p}_{N}\right) \mid \boldsymbol{p}_{i}=\left(\mathcal{A}_{i}, \mu_{i}, \nu_{i}\right) \in \mathbb{R}^{3}, \mathcal{A}_{i}, \nu_{i}>0,\right. \\
\left.i \in \mathcal{N}, \sum_{i \in \mathcal{N}} \mathcal{A}_{i}=\mathcal{A}, \sum_{i \in \mathcal{N}} \tilde{\mu}_{i}=\mathcal{E}\right\}
\end{array}
$$

where $\tilde{\mu}_{i}$ is given in (16), the region $\mathcal{R}_{T}\left(\boldsymbol{p}_{1}, \cdots, \boldsymbol{p}_{N}\right)$ described by the set of $\left(R_{1}, \cdots, R_{N}\right) \in \mathbb{R}_{+}^{N}$ bounded by

$$
R_{i} \leq \frac{1}{2} \log \left(\frac{\nu_{i}^{2}}{\tilde{\nu}_{i}^{2}}+\frac{\nu_{i}^{2}}{\sum_{j=1}^{i-1} \tilde{\nu}_{j}^{2}+\sigma_{i}^{2}}\right)-\phi_{i}, \quad i \in \mathcal{N},
$$

is achievable, where $\phi_{i}=\log \left(\eta_{i}\right)+$ $\frac{1}{2}\left(\left(\mathcal{A}_{i}-\mu_{i}\right) \tilde{g}_{i}\left(\mathcal{A}_{i}\right)+\mu_{i} \tilde{g}_{i}(0)\right), \tilde{g}_{i}\left(x_{i}\right)$ is the distribution function of a $\left(\mathcal{A}_{i}, \mu_{i}, \nu_{i}\right)$-TG random variable, and $\tilde{\nu}_{i}$ is its standard deviation (17). The capacity region of the $N$-user OBC thus satisfies $\mathcal{C} \supseteq \underline{\mathfrak{e}}_{T}=\bigcup_{\left(\boldsymbol{p}_{1}, \cdots, \boldsymbol{p}_{N}\right) \in \mathcal{P}} \mathcal{R}_{T}\left(\boldsymbol{p}_{1}, \cdots, \boldsymbol{p}_{N}\right)$.

Proof: The transmitter sends $X=X_{1}+X_{2}+\cdots+X_{N}$ where $X_{i}$ follows a $\left(\mathcal{A}_{i}, \mu_{i}, \nu_{i}\right)$-TG distribution $\tilde{g}_{i}\left(x_{i}\right)$ as defined in (15). User $i$ decodes $X_{N}, X_{N-1}, \cdots, X_{i}$ in this order. The derivation of the achievable rates is similar to Theorem 2.

Proposition 2: The region $\underline{\mathcal{C}}_{T}^{\prime}=\bigcup_{\boldsymbol{\beta} \in \mathcal{S}_{\boldsymbol{\beta}}} \mathcal{R}_{T}^{\prime}(\boldsymbol{\beta})$ is achievable, where $\mathcal{R}_{T}^{\prime}(\boldsymbol{\beta})$ is the set of rate tuples $\left(R_{1}, \cdots, R_{N}\right) \in$ $\mathbb{R}_{+}^{N}$ satisfying

$$
R_{i} \leq \frac{1}{2} \log \left(1+\frac{\alpha^{2} \beta_{i}^{2} \mathcal{A}^{2}\left(1+\epsilon_{\mu}\right)^{-2}}{\sum_{j=1}^{i-1} \alpha^{2} \beta_{j}^{2} \mathcal{A}^{2}+9 \sigma_{i}^{2}}\right)-\epsilon_{\phi},
$$

$i \in \mathcal{N}$, with $\epsilon_{\phi}=0.016, \epsilon_{\mu}=0.0015, \boldsymbol{\beta}=\left(\beta_{1}, \cdots, \beta_{N}\right)$, and $\mathcal{S}_{\boldsymbol{\beta}}=\left\{\boldsymbol{\beta} \in[0,1]^{N} \mid \sum_{i \in \mathcal{N}} \beta_{i}=1\right\}$.

Proof: Follows from Theorem 6 using $\mathcal{A}_{i}=\beta_{i} \mathcal{A}$ where $\sum_{i \in \mathcal{N}} \beta_{i}=1$, and choosing $\mu_{i}$ and $\nu_{i}=\frac{\mu_{i}}{3}$ such that $\tilde{\mu}_{i}=\alpha \mathcal{A}_{i}$. The derivation of the achievable rates is similar to Proposition 1.

Next, we extend the achievable inner bound using the discrete input distribution to the $N$-user case. Let $X=$ $X_{1}+X_{2}+\cdots+X_{N}$, where $X_{i} \in\left[0, \mathcal{A}_{i}\right]$ has mean $\mathcal{E}_{i}=\alpha \mathcal{A}_{i}$ and distribution

$$
p_{X_{i}}^{*}\left(x_{i}\right)=\sum_{k=0}^{K_{i}} a_{i k} \delta\left(x-k \ell_{i}\right),
$$

with $a_{i k}=\frac{t_{i}^{k}}{\sum_{j=0}^{K_{i}} t_{i}^{j}}$ for $k=0, \cdots, K_{i}, K_{i} \in \mathbb{N}_{+}, \ell_{i}=$ $\frac{\mathcal{A}_{i}}{K_{i}}$, and $t_{i} \in[0,1]$ is the solution of $\sum_{k=0}^{K_{i}}\left(1-\frac{k \mathcal{A}_{i}}{K_{i} \varepsilon_{i}}\right) t^{k}=$ 
0 . Furthermore, $\sum_{i \in \mathcal{N}} \mathcal{A}_{i}=\mathcal{A}$. User $i$ decodes the signals $X_{N}, \cdots, X_{i}$ successively in this order. The achievable rate region is given as follows.

Theorem 7: For $\left(\boldsymbol{q}_{1}, \cdots, \boldsymbol{q}_{N}\right) \in \mathcal{Q}$ defined as

$$
\begin{array}{r}
\mathcal{Q}=\left\{\left(\boldsymbol{q}_{1}, \cdots, \boldsymbol{q}_{N}\right) \mid \boldsymbol{q}_{i}=\left(\mathcal{A}_{i}, K_{i}\right) \in \mathbb{R}_{+} \times \mathbb{N}_{+}, i \in \mathcal{N},\right. \\
\left.\sum_{i \in \mathcal{N}} \mathcal{A}_{i}=\mathcal{A}\right\}
\end{array}
$$

the region $\mathcal{R}_{D}\left(\boldsymbol{q}_{1}, \cdots, \boldsymbol{q}_{N}\right)$ described by the set of $\left(R_{1}, \cdots, R_{N}\right) \in \mathbb{R}_{+}^{N}$ bounded by

$$
\begin{aligned}
R_{N} & \leq I\left(X_{N} ; Y_{N}\right) \\
R_{i} & \leq I\left(X_{i} ; Y_{i} \mid X_{i+1}, \cdots, X_{N}\right), \quad i \in \mathcal{N} \backslash\{N\},
\end{aligned}
$$

is achievable, where $X_{i}$ follows the distribution $p_{X_{i}}^{*}$ defined in (44). The capacity region $\mathcal{C}$ of the $N$-user OBC thus satisfies $\mathcal{C} \supseteq \underline{\mathcal{C}}_{D}=\bigcup_{\left(q_{1}, \cdots, q_{N}\right) \in \mathcal{Q}} \mathcal{R}_{D}\left(\boldsymbol{q}_{1}, \cdots, \boldsymbol{q}_{N}\right)$.

Proof: Using superposition coding as in [24], [26] with the distribution $p_{X_{i}}^{*}(44)$.

\section{A. High SNR Analysis}

By replacing the upper bound $\bar{C}_{\alpha}^{[j]}(\mathcal{A}, \sigma)$ in Theorem 5 by $\bar{C}_{\alpha}^{[h]}(\mathcal{A}, \sigma)(8)$, we obtain the following high-SNR outer bound for the $N$-user OBC.

Corollary 5: The high-SNR capacity region of the $N$ user OBC is outer bounded by $\overline{\mathcal{C}}^{[h]}=\bigcup_{\boldsymbol{\rho} \in \mathcal{S}_{\rho}} \overline{\mathcal{R}}^{[h]}(\boldsymbol{\rho})$ where $\overline{\mathcal{R}}^{[h]}(\boldsymbol{\rho})$ is the set of $\left(R_{1}, \cdots, R_{N}\right) \in \mathbb{R}_{+}^{N}$ satisfying

$$
R_{i} \leq \frac{1}{2} \log \left(\frac{\sigma_{i}^{2}+c \rho_{i+1}^{2} \mathcal{A}^{2}}{\sigma_{i}^{2}+c \rho_{i}^{2} \mathcal{A}^{2}}\right), \quad i \in \mathcal{N},
$$

with $\mathcal{S}_{\boldsymbol{\rho}}=\left\{\left(\rho_{1}, \cdots, \rho_{N}\right) \in[0,1]^{N} \mid \rho_{i} \leq \rho_{i+1} \forall i \in \mathcal{N}, \rho_{1}=\right.$ $\left.0, \rho_{N+1}=1\right\}$ and $c$ as given in (9).

Proof: Follows from Theorem 5 and the upper bound (8).

Using this corollary, we can bound the high-SNR symmetric-capacity of the $N$-user OBC as follows.

Corollary 6: At high SNR, the symmetric-capacity of the $N$-user OBC satisfies $\underline{C}_{h} \leq C_{h} \leq \bar{C}_{h}$, where $\underline{C}_{h}=$ $\frac{1}{2} \log \left(1+\frac{1}{2} \sqrt[N]{\frac{2 \alpha^{2} \mathcal{A}^{2}}{9 N^{2} \sigma_{1}^{2}}}\right)$ and $\bar{C}_{h}=\frac{1}{2} \log \left(1+\sqrt[N]{\frac{c \mathcal{A}^{2}}{\sigma_{1}^{2}}}\right)$. Furthermore, $\bar{C}_{h}-\underline{C}_{h} \stackrel{\frac{\varepsilon}{\sigma_{N}} \rightarrow \infty}{\longrightarrow} \frac{1}{2} \log \left(2 \sqrt[N]{\frac{9 c N^{2}}{2 \alpha^{2}}}\right) \leq$ $\frac{1}{2} \log \left(2 \sqrt[N]{\frac{9 e N^{2}}{4 \pi}}\right)$ nats/transmission.

Proof: See Appendix C.

The maximum gap $\frac{1}{2} \log \left(2 \sqrt[N]{\frac{9 e N^{2}}{4 \pi}}\right)$ decreases in $N$ from 0.86 nats for $N=2$ to $\frac{1}{2} \log (2)$ nats $\left(\frac{1}{2}\right.$ bits) for large $N$. Corollary 2 provides a tighter characterization for $N=2$. The high-SNR capacity region of the $N$-user OBC can be bounded as given next.

Corollary 7: At high SNR, for the $N$-user OBC, we have $\underline{\mathrm{e}}^{[h]} \subset \mathcal{C} \subseteq \overline{\mathrm{e}}^{[h]}$, where $\overline{\mathrm{e}}^{[h]}=\left\{\left(R_{1}, \cdots, R_{N}\right) \in\right.$ $\left.\mathbb{R}_{+}^{N} \mid\left(R_{1}, \cdots, R_{N-1}, R_{N}+\delta\right) \in \overline{\mathcal{C}}^{[h]}\right\}$ with $\delta=\log \left(\frac{3 \sqrt{c N}}{\alpha}\right)$ and $\overline{\mathcal{C}}^{[h]}$ as in Corollary 5. Furthermore, $\delta \leq 0.68+\frac{1}{2} \log (N)$ nats/transmission.
Proof: See Appendix D.

Note that the gap here is in one direction only, namely, in the $R_{N}$ direction. If we fix a tuple $\boldsymbol{R}=\left(R_{1}, \cdots, R_{N-1}, R_{N}+\right.$ $\delta$ ) on the boundary of $\overline{\mathcal{C}}^{[h]}$, and choose an achievable rate tuple with back-off $\xi_{i}$ from $\boldsymbol{R}$ in the $i$-th direction, $i \in \mathcal{N} \backslash$ $\{N\}$, then the achievable rate of the $N$-th user becomes higher than $R_{N}$. The achievable rate in this case can be expressed as $\left(R_{1}-\xi_{1}, \cdots, R_{N-1}-\xi_{N-1}, R_{N}+\xi_{N}\right)$. Roughly speaking, this distributes the gap $\delta$ between the $N$ directions, so that the gap per user becomes proportional to $\frac{1}{N} \log (N)$. The gap here is bounded without using time-sharing in combination with the inner bound in Proposition 2. Hence, this gap can be reduced by incorporating time-sharing in a manner similar to the proof of Corollary 3. Next, we consider the low SNR regime.

\section{B. Low SNR Analysis}

Using the upper bound $\bar{C}_{\alpha}^{[3]}(\mathcal{A}, \sigma)$ given in Lemma 1 in conjunction with Theorem 5 , we can obtain a simple outer bound on $\mathcal{C}$ which is tight at low SNR. This is stated next.

Theorem 8: The capacity region of the $N$-user OBC asymptotically coincides at low SNR with the region

$$
\widehat{\mathcal{C}}^{[3]}=\left\{\left(R_{1}, \cdots, R_{N}\right) \in \mathbb{R}_{+}^{N} \mid \sum_{i \in \mathcal{N}} \sigma_{i}^{2} R_{i} \leq \frac{\alpha(1-\alpha) \mathcal{A}^{2}}{2}\right\},
$$

which is achievable by OOK combined with TDMA.

Proof: Using Theorem 5 and the upper bound $\bar{C}_{\alpha}^{[3]}(\mathcal{A}, \sigma)$ in Lemma 1, we obtain the outer bound $\overline{\mathcal{C}}^{[3]}=\bigcup_{\boldsymbol{\rho} \in \mathcal{S}_{\boldsymbol{\rho}}} \overline{\mathcal{R}}^{[3]}(\boldsymbol{\rho})$ where $\overline{\mathcal{R}}^{[3]}(\boldsymbol{\rho})$ is the set of $\left(R_{1}, \cdots, R_{N}\right) \in \mathbb{R}_{+}^{N}$ that satisfy

$$
R_{i} \leq \frac{1}{2} \log \left(\frac{\sigma_{i}^{2}+\rho_{i+1}^{2} \alpha(1-\alpha) \mathcal{A}^{2}}{\sigma_{i}^{2}+\rho_{i}^{2} \alpha(1-\alpha) \mathcal{A}^{2}}\right), \quad \forall i \in \mathcal{N},
$$

for $\boldsymbol{\rho}=\left(\rho_{1}, \cdots, \rho_{N}\right)$, and $\mathcal{S}_{\boldsymbol{\rho}}=\left\{\boldsymbol{\rho} \in[0,1]^{N} \mid \rho_{i} \leq \rho_{i+1} \forall i \in\right.$ $\left.\mathcal{N}, \rho_{1}=0, \rho_{N+1}=1\right\}$. By defining $\gamma_{i}^{2}=\rho_{i+1}^{2}-\rho_{i}^{2}$, we write (47) as follows

$$
R_{i} \leq \frac{1}{2} \log \left(1+\frac{\gamma_{i}^{2} \alpha(1-\alpha) \mathcal{A}^{2}}{\sigma_{i}^{2}+\sum_{j=0}^{i-1} \gamma_{j}^{2} \alpha(1-\alpha) \mathcal{A}^{2}}\right),
$$

$i \in \mathcal{N}$, where $\gamma_{0}=0$ and $\sum_{i \in \mathcal{N}} \gamma_{i}^{2}=1$. This rate constraint can be relaxed to $R_{i} \leq \frac{\gamma_{i}^{2} \alpha(1-\alpha) \mathcal{A}^{2}}{2 \sigma_{i}^{2}}$ using $\log (1+x) \leq x$ and $\frac{a}{b+c} \leq \frac{a}{b}$ for positive $a, b$, and $c$, leading to an outer bound given by $\widehat{\mathrm{e}}^{[3]}$ in the theorem. The boundary of $\widehat{\mathcal{C}}^{[3]}$ is the plane defined by $\sum_{i \in \mathcal{N}} \sigma_{i}^{2} R_{i}=\frac{\alpha(1-\alpha) \mathcal{A}^{2}}{2}$ for $R_{i} \geq$ 0 . This plane intersects with the $R_{i}$-axis at $R_{i}=\frac{\alpha(1-\alpha) \mathcal{A}^{2}}{2 \sigma_{i}^{2}}$. Let the rate achievable by using OOK to send information to user $i$ only be denoted $R_{\alpha}\left(\mathcal{A}, \sigma_{i}\right)$. We know from [9] that $R_{\alpha}\left(\mathcal{A}, \sigma_{i}\right) \stackrel{\frac{\mathcal{A}}{\sigma_{1}} \rightarrow 0}{\longrightarrow} \frac{\alpha(1-\alpha) \mathcal{A}^{2}}{2 \sigma_{i}^{2}}$. Thus, the corners of the outer bound are achievable. Since the boundary of this outer bound is a plane, it can be achieved by time-sharing between its $N$ corners with time-sharing parameters $\gamma_{i}^{2}$. This proves the achievability of $\widehat{\mathcal{C}}^{[3]}$ and completes the proof.

This theorem leads to the low-SNR symmetric capacity of the $N$-user OBC, as follows.

Corollary 8: The symmetric-capacity of the $N$-user OBC satisfies $C_{l} \stackrel{\frac{\mathcal{A}}{\sigma_{1}} \rightarrow 0}{\longrightarrow} \frac{\alpha(1-\alpha) \mathcal{A}^{2}}{2 \sum_{i=1}^{N} \sigma_{i}^{2}}$. 


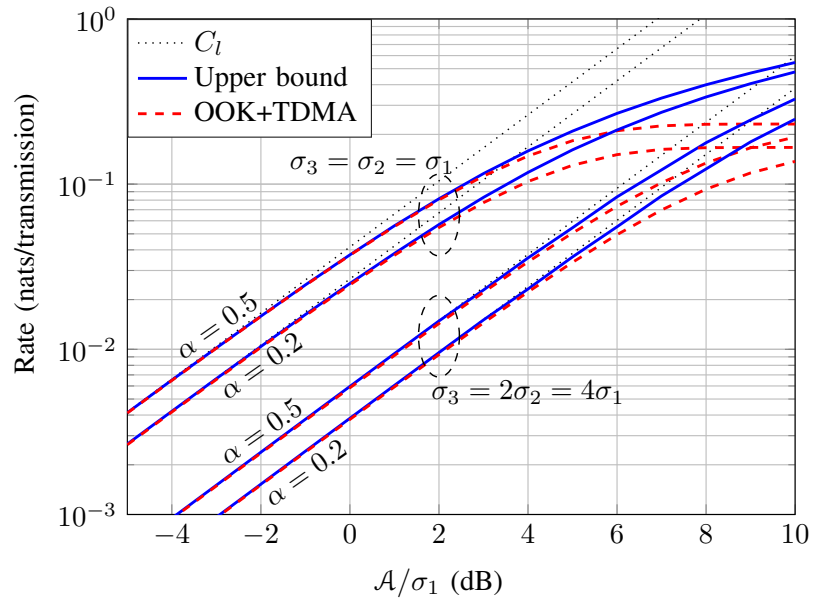

Fig. 9: Symmetric capacity bounds versus $\frac{\mathcal{A}}{\sigma_{1}}$ for a 3 -user $\mathrm{OBC}$.

Proof: By finding the point $\left(R_{1}, \cdots, R_{N}\right)$ satisfying $R_{i}=R$ for all $i \in \mathcal{N}$, which lies on the boundary of $\widehat{\mathcal{C}}^{[3]}$ in Theorem 8.

Fig. 9 shows symmetric-capacity upper and lower bounds for a 3-user OBC. The upper bound is obtained by finding the intersection of the outer bound $\overline{\mathcal{C}}^{[3]}$ given in (47) with the line $R_{3}=R_{2}=R_{1}$. The lower bound is obtained by a combination of OOK and TDMA, i.e., by solving $\tau_{i} R_{\alpha}\left(\mathcal{A}, \sigma_{i}\right)=\tau_{j} R_{\alpha}\left(\mathcal{A}, \sigma_{j}\right), i, j \in\{1,2,3\}, i \neq j$, for $\tau_{i}$, such that $\tau_{1}+\tau_{2}+\tau_{3}=1$, where $R_{\alpha}\left(\mathcal{A}, \sigma_{i}\right)$ is the rate achieved by using OOK to send to user $i$ only. At low-SNR, the symmetric-capacity achieving time-sharing parameters are $\tau_{i}=\gamma_{i}^{2}$ with $\gamma_{i}^{2}=\frac{\sigma_{i}^{2}}{\sum_{j=1}^{N} \sigma_{j}^{2}}$, which follows by solving $\frac{\gamma_{i}^{2} \alpha(1-\alpha) \mathcal{A}^{2}}{2 \sigma_{i}^{2}}=\frac{\gamma_{j}^{2} \alpha(1-\alpha) \mathcal{A}^{2}}{2 \sigma_{j}^{2}}$ for all $i, j \in \mathcal{N}, i \neq j$. In particular, for $N=3$, we have $\tau_{i}=\frac{\sigma_{i}^{2}}{\sigma_{1}^{2}+\sigma_{2}^{2}+\sigma_{3}^{2}}$. Note the convergence of the bounds at low SNR in Fig. 9.

This concludes the high and low SNR analysis of the $N$ user OBC. It remains to say that at moderate SNR, the discrete input distribution performs better than the TG distribution.

\section{CONCLUSION}

In this paper, we studied the capacity of the IM-DD optical broadcast channel (OBC). For this channel, we have derived capacity region outer and inner bounds. The outer bounds are derived by combining existing upper bounds on the capacity of the IM-DD P2P channel and Bergmans' approach. The inner bounds are achieved using superposition coding and either truncated-Gaussian distributions or discrete input distributions.

We have shown that a superposition of truncated-Gaussian inputs achieves the capacity region within a constant gap at high SNR. We have also shown that as far as the symmetriccapacity is concerned, time-sharing between superposition coding strategies is not necessary at high SNR. On the other hand, we have shown that on-off keying (OOK) combined with TDMA is optimal at low SNR. This is particularly interesting since both OOK and TDMA are practically simple to implement. Thus, in an office environment with strong noise for instance (strong background radiation or noisy equipment), the optimal scheme is simply OOK with TDMA. For moderate SNR, a discrete input distribution is better than truncatedGaussian.

As an extension of this work, it would be interesting to study the impact of fading on the capacity of the OBC. This can be studied using frameworks similar to [27]-[29]. Another important aspect is secrecy since broadcasting is vulnerable to eavesdropping. As such, an interesting direction for future work is investigating secure broadcast and optical wiretap channels.

\section{APPENDIX A \\ BOUNDING $\tilde{\mu}, \tilde{\nu}$, AND $\phi$ FOR $\nu=\frac{\mu}{3}$}

Consider a $(\mathcal{A}, \mu, \nu)$-TG distribution $\tilde{g}(x)$ with $\nu=\frac{\mu}{3}$ and $0 \leq \mu \leq \frac{\mathcal{A}}{2}$. Its mean is

$$
\begin{aligned}
\tilde{\mu} & =\nu^{2}(\tilde{g}(0)-\tilde{g}(\mathcal{A}))+\mu \\
& =\frac{\eta \nu}{\sqrt{2 \pi}}\left(e^{-\frac{\mu^{2}}{2 \nu^{2}}}-e^{-\frac{(\mathcal{A}-\mu)^{2}}{2 \nu^{2}}}\right)+\mu,
\end{aligned}
$$

where $\eta=\left(G_{\mu, \nu}(\mathcal{A})-G_{\mu, \nu}(0)\right)^{-1}$. Since $e^{x}>0$, we have $\tilde{\mu}<\frac{\eta \nu}{\sqrt{2 \pi}} e^{-\frac{\mu^{2}}{2 \nu^{2}}}+\mu=\frac{\eta}{3 \sqrt{2 \pi e^{9}}} \mu+\mu$. Moreover, since $\mu \leq \frac{\mathcal{A}}{2}$, then $G_{\mu, \nu}(\mathcal{A})-G_{\mu, \nu}(0)>G_{\mu, \nu}(2 \mu)-G_{\mu, \nu}(0)>0.997$, which implies that $\eta<1.0027$. Therefore, $\tilde{\mu}<\mu(1+0.0015)$. On the other hand, clearly for $\mu \leq \frac{\mathcal{A}}{2}, \mu \leq \tilde{\mu}$, and hence $\mu \leq \tilde{\mu}<\mu(1+0.0015)$. Similarly we can bound the variance $\tilde{\nu}^{2}$ given by (17) as

$$
\tilde{\nu}^{2}=\nu^{2}\left(1-(\mathcal{A}-\mu) \tilde{g}(\mathcal{A})-\mu \tilde{g}(0)-\nu^{2}(\tilde{g}(0)-\tilde{g}(\mathcal{A}))^{2}\right) .
$$

First note that $\tilde{g}(0) \geq \tilde{g}(\mathcal{A})$ for $\mu \leq \frac{\mathcal{A}}{2}$. Thus, $\nu^{2}(\tilde{g}(0)-\tilde{g}(\mathcal{A}))^{2} \leq(\nu \tilde{g}(0))^{2}=\frac{\eta^{2}}{2 \pi e^{9}}$. Moreover, since the function $x e^{-x^{2}}$ is decreasing for $x>\frac{1}{\sqrt{2}}$, and since $\mathcal{A}-\mu \geq \mu$ for $\mu \leq \frac{\mathcal{A}}{2}$, we have $(\mathcal{A}-\mu) \tilde{g}(\mathcal{A}) \leq \mu \tilde{g}(0)=\frac{3 \eta}{\sqrt{2 \pi e^{9}}}$. Thus, $\tilde{\nu}^{2} \geq \nu^{2}\left(1-\frac{6 \eta}{\sqrt{2 \pi e^{9}}}-\frac{\eta^{2}}{2 \pi e^{9}}\right)=0.9733 \nu^{2}$. Furthermore, since $\mathcal{A} \geq \mu \geq 0$, then $\tilde{\nu}^{2} \leq \nu^{2}$, and hence $(1-0.0267) \nu^{2} \leq$ $\tilde{\nu}^{2} \leq \nu^{2}$. Finally,

$$
\phi=\log (\eta)+\frac{1}{2}((\mathcal{A}-\mu) \tilde{g}(\mathcal{A})+\mu \tilde{g}(0)),
$$

can be bounded using $(\mathcal{A}-\mu) \tilde{g}(\mathcal{A}) \leq \mu \tilde{g}(0)=\frac{3 \eta}{\sqrt{2 \pi e^{9}}}$ and $\eta<1.0027$ to obtain $\phi \leq 0.016$.

\section{APPENDIX B}

\section{OUTER BOUND FOR THE $N$-USER OBC}

Here, we prove Theorem 5. Similar to the proof of Theorem 1 , we can write

$$
\begin{aligned}
& h\left(\boldsymbol{Y}_{N} \mid W_{N}\right) \\
& =n \overline{\mathrm{e}_{\alpha}^{[j]}}\left(\rho_{N} \mathcal{A}, \sigma_{N}\right)+n \log \left(\sqrt{2 \pi e} \sigma_{N}\right) \\
& =\frac{n}{2} \log \left(2 \pi e \sigma_{N}^{2}+2 \pi e \sigma_{N}^{2}\left(e^{2 \overline{\mathrm{e}}_{\alpha}^{[j]}\left(\rho_{N} \mathcal{A}, \sigma_{N}\right)}-1\right)\right),
\end{aligned}
$$


for some $\rho_{N} \in[0,1]$. On the other hand,

$$
\begin{aligned}
h\left(\boldsymbol{Y}_{N}\right) & \leq n \overline{\mathrm{C}}_{\alpha}^{[j]}\left(\mathcal{A}, \sigma_{N}\right)+n \log \left(\sqrt{2 \pi e} \sigma_{N}\right) \\
& =\frac{n}{2} \log \left(2 \pi e \sigma_{N}^{2}+2 \pi e \sigma_{N}^{2}\left(e^{2 \overline{\mathrm{C}}_{\alpha}^{[j]}\left(\mathcal{A}, \sigma_{N}\right)}-1\right)\right) .
\end{aligned}
$$

Therefore, we can upper bound $R_{N}$ as given in (41). Now we start an induction to bound $R_{i}$ for $i \in \mathcal{N} \backslash\{N\}$. Assume that $h\left(\boldsymbol{Y}_{i+1} \mid W_{i+1}, \cdots, W_{N}\right)$ can be written similar to (52) as

$$
\begin{aligned}
& h\left(\boldsymbol{Y}_{i+1} \mid W_{i+1}, \cdots, W_{N}\right) \\
& =\frac{n}{2} \log \left(2 \pi e \sigma_{i+1}^{2}+2 \pi e \sigma_{N}^{2}\left(e^{2 \overline{\mathrm{e}}_{\alpha}^{[j]}\left(\rho_{i+1} \mathcal{A}, \sigma_{N}\right)}-1\right)\right),
\end{aligned}
$$

for some $i \in \mathcal{N} \backslash\{N\}$, and some $\rho_{i+1} \in[0,1]$. Note that this is true for $i=N-1$. Then, we have

$$
\begin{aligned}
h\left(\boldsymbol{Y}_{i} \mid W_{i}, \cdots, W_{N}\right) & \geq h\left(\boldsymbol{Y}_{i} \mid W_{i} \cdots, W_{N}, \boldsymbol{X}\right) \\
& =h\left(\boldsymbol{Z}_{i}\right) \\
& =n \log \left(\sqrt{2 \pi e} \sigma_{i}\right)
\end{aligned}
$$

and

$$
\begin{aligned}
& h\left(\boldsymbol{Y}_{i} \mid W_{i}, \cdots, W_{N}\right) \\
& \leq h\left(\boldsymbol{Y}_{i} \mid W_{i+1}, \cdots, W_{N}\right) \\
& \leq \frac{n}{2} \log \left(e^{\frac{2}{n} h\left(\boldsymbol{Y}_{i+1} \mid W_{i+1}, \cdots, W_{N}\right)}-2 \pi e\left(\sigma_{i+1}^{2}-\sigma_{i}^{2}\right)\right) \\
& =\frac{n}{2} \log \left(2 \pi e \sigma_{i}^{2}+2 \pi e \sigma_{N}^{2}\left(e^{2 \overline{\mathrm{C}}_{\alpha}^{[j]}\left(\rho_{i+1} \mathcal{A}, \sigma_{N}\right)}-1\right)\right),
\end{aligned}
$$

where the first step follows since conditioning reduces entropy and the second by using the EPI with $\sigma_{i+1}^{2} \geq \sigma_{i}^{2}$. Thus, we can write

$$
\begin{aligned}
& h\left(\boldsymbol{Y}_{i} \mid W_{i}, \cdots, W_{N}\right) \\
& =\frac{n}{2} \log \left(2 \pi e \sigma_{i}^{2}+2 \pi e \sigma_{N}^{2}\left(e^{2 \overline{\mathrm{e}}_{\alpha}^{[j]}\left(\rho_{i} \mathcal{A}, \sigma_{N}\right)}-1\right)\right),
\end{aligned}
$$

for some $\rho_{i} \in\left[0, \rho_{i+1}\right]$. Using this equality, we can bound $h\left(\boldsymbol{Y}_{i} \mid W_{i+1}, \cdots, W_{N}\right)$ as follows

$$
\begin{aligned}
& h\left(\boldsymbol{Y}_{i} \mid W_{i+1}, \cdots, W_{N}\right) \\
& \leq \frac{n}{2} \log \left(e^{\frac{2}{n} h\left(\boldsymbol{Y}_{i+1} \mid W_{i+1}, \cdots, W_{N}\right)}-e^{\frac{2}{n} h\left(\tilde{\boldsymbol{Z}}_{i+1} \mid W_{i+1}, \cdots, W_{N}\right)}\right) \\
& =\frac{n}{2} \log \left(e^{\frac{2}{n} h\left(\boldsymbol{Y}_{i+1} \mid W_{i+1}, \cdots, W_{N}\right)}-2 \pi e\left(\sigma_{i+1}^{2}-\sigma_{i}^{2}\right)\right) \\
& =\frac{n}{2} \log \left(2 \pi e \sigma_{i}^{2}+2 \pi e \sigma_{N}^{2}\left(e^{2 \bar{C}_{\alpha}^{[j]}\left(\rho_{i+1} \mathcal{A}, \sigma_{2}\right)}-1\right)\right),
\end{aligned}
$$

where the first step follows by writing $\boldsymbol{Y}_{i+1}=\boldsymbol{Y}_{i}+\tilde{\boldsymbol{Z}}_{i+1}$ with $Z_{i+1}=Z_{i}+\tilde{Z}_{i+1}$ and $\tilde{Z}_{i+1} \sim g_{0, \sqrt{\sigma_{i+1}^{2}-\sigma_{i}^{2}}}\left(\tilde{z}_{2}\right)$ independent of $Z_{i}$, and by using the conditional EPI, and the last step follows by applying (53). Now we can write the following bound on $R_{i}$

$$
\begin{aligned}
n\left(R_{i}-\varepsilon_{i n}\right) & \leq I\left(W_{i} ; \boldsymbol{Y}_{i}\right) \\
& \leq I\left(W_{i} ; \boldsymbol{Y}_{i} \mid W_{i+1}, \cdots, W_{N}\right) \\
& \leq h\left(\boldsymbol{Y}_{i} \mid W_{i+1}, \cdots, W_{N}\right)-h\left(\boldsymbol{Y}_{i} \mid W_{i}, \cdots, W_{N}\right),
\end{aligned}
$$

which follows similar to the proof of Theorem 1. Substituting (60) and (62) in this last inequality and letting $n \rightarrow \infty$ yields the desired bound on $R_{i}$. Finally, by taking the union over all $\rho_{i} \in[0,1]$ with $\rho_{i} \leq \rho_{i+1}$, we get the statement of the Theorem 5. Note that we can set $\rho_{1}=0$ since $h\left(\boldsymbol{Y}_{1} \mid W_{1}, \cdots, W_{N}\right) \geq h\left(\boldsymbol{Y}_{1} \mid W_{1}, \cdots, W_{N}, \boldsymbol{X}\right)=$ $n \log \left(\sqrt{2 \pi e} \sigma_{1}\right)$.

\section{APPENDIX C}

\section{SYMMETRIC-CAPACITY BOUNDS FOR THE $N$-USER OBC}

To prove Corollary 6, we start by deriving a symmetriccapacity upper bound. From Corollary 5, we have $R_{i} \leq$ $\frac{1}{2} \log \left(\frac{\sigma_{i}^{2}+c \rho_{i+1}^{2} \mathcal{A}^{2}}{\sigma_{i}^{2}+c \rho_{i}^{2} \mathcal{A}^{2}}\right)$, for all $i \in \mathcal{N}$, where $0 \leq \rho_{i} \leq \rho_{i+1} \leq 1$, $\rho_{1}=0$ and $\rho_{N+1}=1$. For convenience, we write this bounds as

$$
R_{i} \leq \frac{1}{2} \log \left(1+\frac{c \gamma_{i}^{2} \mathcal{A}^{2}}{\sigma_{i}^{2}+c \sum_{j=0}^{i-1} \gamma_{j}^{2} \mathcal{A}^{2}}\right), \quad i \in \mathcal{N},
$$

where $\gamma_{i}^{2}=\rho_{i+1}^{2}-\rho_{i}^{2}, \gamma_{0}=0$, and $\sum_{i \in \mathcal{N}} \gamma_{i}^{2}=1$. Thus, we have $R_{1} \leq \frac{1}{2} \log \left(1+\frac{c \gamma_{1}^{2} \mathcal{A}^{2}}{\sigma_{1}^{2}}\right)$. In the remaining rate constraints, we will neglect $\sigma_{i}^{2}$ in comparison to $c \sum_{j=0}^{i-1} \gamma_{j}^{2} \mathcal{A}^{2}$ since we are focusing on the high SNR regime. Thus for those rates, we have $R_{i} \leq \frac{1}{2} \log \left(1+\frac{\gamma_{i}^{2}}{\sum_{j=0}^{i-1} \gamma_{j}^{2}}\right)$ for $i \in \mathcal{N} \backslash\{1\}$. To find an upper bound on the symmetric-rate, we have to equate the rate constraints for all $i \in \mathcal{N}$. This yields $\frac{\gamma_{1}^{2}}{\gamma^{2}}=\frac{\gamma_{i}^{2}}{\sum_{j=0}^{i-1} \gamma_{j}^{2}}$ $\forall i \in \mathcal{N} \backslash\{1\}$, where $\gamma^{2}=\frac{\sigma_{1}^{2}}{c \mathcal{A}^{2}}$. The solution of this system gives

$$
\gamma_{i}^{2}=\frac{\gamma_{1}^{2}}{\gamma^{2}}\left(1+\frac{\gamma_{1}^{2}}{\gamma^{2}}\right)^{i-2} \gamma_{1}^{2} .
$$

Substituting in $\sum_{i \in \mathcal{N}} \gamma_{i}^{2}=1$ leads to $f\left(\gamma_{1}^{2}\right)=1$ where

$$
f\left(\gamma_{1}^{2}\right)=\gamma_{1}^{2}\left(1+\frac{\gamma_{1}^{2}}{\gamma^{2}} \sum_{i=2}^{N}\left(1+\frac{\gamma_{1}^{2}}{\gamma^{2}}\right)^{i-2}\right) .
$$

By solving this expression for $\frac{\gamma_{1}^{2}}{\gamma^{2}}$ and substituting in $R_{1} \leq$ $\frac{1}{2} \log \left(1+\frac{\gamma_{1}^{2}}{\gamma^{2}}\right)$, we get a symmetric-capacity upper bound. Instead of solving this equation for $\frac{\gamma_{1}^{2}}{\gamma^{2}}$, we upper bound the solution. First, we note that $f\left(\gamma_{1}^{2}\right)$ is increasing in $\frac{\gamma_{1}^{2}}{\gamma^{2}}$. Thus, by lower bounding $f\left(\gamma_{1}^{2}\right)$ by some function $f_{0}\left(\gamma_{1}^{2}\right)$ and solving $f_{0}\left(\gamma_{1}^{2}\right)=1$, we get an upper bound on the solution of $f\left(\gamma_{1}^{2}\right)=1$. To this end, we lower bound $f\left(\gamma_{1}^{2}\right)$ as follows

$$
\begin{aligned}
f\left(\gamma_{1}^{2}\right) & >\gamma_{1}^{2}\left(1+\frac{\gamma_{1}^{2}}{\gamma^{2}} \sum_{i=2}^{N}\left(\frac{\gamma_{1}^{2}}{\gamma^{2}}\right)^{i-2}\right) \\
& =\gamma^{2} \sum_{i=1}^{N}\left(\frac{\gamma_{1}^{2}}{\gamma^{2}}\right)^{i} \\
& >\gamma^{2}\left(\frac{\gamma_{1}^{2}}{\gamma^{2}}\right)^{N} \\
& =f_{0}\left(\gamma_{1}^{2}\right) .
\end{aligned}
$$

Setting $f_{0}\left(\gamma_{1}^{2}\right)=1$ yields $\frac{\gamma_{1}^{2}}{\gamma^{2}}=\sqrt[N]{\frac{1}{\gamma^{2}}}$. Therefore, the highSNR symmetric-capacity upper bounded by

$$
\bar{C}_{h}=\frac{1}{2} \log \left(1+\sqrt[N]{\frac{c \mathcal{A}^{2}}{\sigma_{1}^{2}}}\right),
$$

which proves the upper bound in Corollary 6 . 
Now we prove the lower bound. Based on Proposition 2, the rates given by

$$
R_{i} \leq \frac{1}{2} \log \left(1+\frac{\alpha^{2} \beta_{i}^{2} \mathcal{A}^{2}\left(1+\epsilon_{\mu}\right)^{-2}}{\sum_{j=1}^{i-1} \alpha^{2} \beta_{j}^{2} \mathcal{A}^{2}+9 \sigma_{i}^{2}}\right)-\epsilon_{\phi},
$$

$i \in \mathcal{N}$, are achievable, where $\beta_{i} \in[0,1]$, and $\sum_{i \in \mathcal{N}} \beta_{i}=$ 1. Let us first neglect $\epsilon_{\phi}$ and $\epsilon_{\mu}$. Then, we neglect $9 \sigma_{i}^{2}$ in comparison to $\sum_{j=1}^{i-1} \alpha^{2} \beta_{j}^{2} \mathcal{A}^{2}$ at high SNR. This yields,

$$
\begin{aligned}
R_{1} & \leq \frac{1}{2} \log \left(1+\frac{\alpha^{2} \beta_{1}^{2} \mathcal{A}^{2}}{9 \sigma_{1}^{2}}\right) \\
R_{i} & \leq \frac{1}{2} \log \left(1+\frac{\beta_{i}^{2}}{\sum_{j=1}^{i-1} \beta_{j}^{2}}\right), \quad i \in \mathcal{N} \backslash\{1\} .
\end{aligned}
$$

To find the achievable symmetric-rate, we have to equate the rate constraints as we have done above. This yields the same solution as (66), with $\gamma_{i}$ replaced by $\beta_{i}$, and $\gamma$ by $\beta=\frac{9 \sigma_{1}^{2}}{\alpha^{2} \mathcal{A}^{2}}$. However, contrary to $\gamma_{i}$, the constraint on $\beta_{i}$ is linear, given by $\sum_{i \in \mathcal{N}} \beta_{i}=1$. Thus, we have $f^{\prime}\left(\beta_{1}\right)=1$ where

$$
f^{\prime}\left(\beta_{1}\right)=\beta_{1}\left(1+\frac{\beta_{1}}{\beta} \sum_{i=2}^{N}\left(1+\frac{\beta_{1}^{2}}{\beta^{2}}\right)^{\frac{i-2}{2}}\right) .
$$

Similar to above, this function is increasing in $\beta_{1}>0$, and thus, a lower bound on the solution of $f^{\prime}\left(\beta_{1}\right)=1$ can be obtained by upper bounding $f^{\prime}\left(\beta_{1}\right)$. To this end, we write

$$
\begin{aligned}
f^{\prime}\left(\beta_{1}\right) & <\beta_{1}\left(1+\frac{\sqrt{2} \beta_{1}}{\beta} \sum_{i=2}^{N}\left(2 \frac{\beta_{1}^{2}}{\beta^{2}}\right)^{\frac{i-2}{2}}\right) \\
& =\frac{\beta}{\sqrt{2}} \sum_{i=1}^{N}\left(\frac{\beta_{1} \sqrt{2}}{\beta}\right)^{i} \\
& <N \frac{\beta}{\sqrt{2}}\left(\frac{\beta_{1} \sqrt{2}}{\beta}\right)^{N} \\
& =f_{0}^{\prime}\left(\beta_{1}\right),
\end{aligned}
$$

where we have chosen $\beta_{1}>\beta$ since choosing $\beta_{1}<\beta$ leads to the trivial zero symmetric-rate. Setting $f_{0}^{\prime}\left(\beta_{1}\right)=1$ yields $\frac{\beta_{1}}{\beta}=\frac{1}{\sqrt{2}} \sqrt[N]{\frac{\sqrt{2}}{N \beta}}$. Recall that this is a lower bound on the solution of $f^{\prime}\left(\beta_{1}\right)=1$. Substituting this lower bound in (74) leads to the symmetric-capacity lower bound

$$
\underline{C}_{h}=\frac{1}{2} \log \left(1+\frac{1}{2} \sqrt[N]{\frac{2 \alpha^{2} \mathcal{A}^{2}}{9 N^{2} \sigma_{1}^{2}}}\right),
$$

which proves the lower bound in Corollary 6 .

With these upper and lower bounds, bounding the gap becomes simple. We calculate the difference $\bar{C}_{h}-\underline{C}_{h}$ after using $\log (1+x) \stackrel{x \rightarrow \infty}{\longrightarrow} \log (x)$, to obtain $\bar{C}_{h}-\underline{C}_{h} \leq$ $\frac{1}{2} \log \left(2 \sqrt[N]{\frac{9 c N^{2}}{2 \alpha^{2}}}\right)$. By substituting the value of $c$, we get the maximum gap $\frac{1}{2} \log \left(2 \sqrt[N]{\frac{9 e N^{2}}{4 \pi}}\right)$ as in Corollary 6 .

\section{APPENDIX D}

\section{CAPACITy Region GaP FOR THE $N$-USER OBC}

Here, we prove Corollary 7. Using (65), we upper bound $R_{N}$ for a given $\left(R_{1}, \cdots, R_{N-1}\right)$ by

$$
R_{N} \leq \frac{1}{2} \log \left(\frac{\sigma_{N}^{2}+c \mathcal{A}^{2}}{\sigma_{N}^{2}+\sum_{i=1}^{N-1} \sigma_{i}^{2}\left(e^{2 R_{i}}-1\right) \prod_{k=i+1}^{N-1} e^{2 R_{k}}}\right) .
$$

Similarly, using (73) (after neglecting $\epsilon_{\phi}$ and $\epsilon_{\nu}$ ), the achievable rate $R_{N}$ for a given achievable $\left(R_{1}, \cdots, R_{N-1}\right)$ is constrained by

$R_{N} \leq \frac{1}{2} \log \left(\frac{9 \sigma_{N}^{2}+\alpha^{2} \sum_{i \in \mathcal{N}} \beta_{i}^{2} \mathcal{A}^{2}}{9 \sigma_{N}^{2}+9 \sum_{i=1}^{N-1} \sigma_{i}^{2}\left(e^{2 R_{i}}-1\right) \prod_{k=i+1}^{N-1} e^{2 R_{k}}}\right)$,

given $\sum_{i \in \mathcal{N}} \beta_{i}=1$. The gap between (82) and $R_{N}$ (83) is thus

$$
\Delta=\frac{1}{2} \log \left(\frac{\sigma_{N}^{2}+c \mathcal{A}^{2}}{\sigma_{N}^{2}+\frac{1}{9} \alpha^{2} \sum_{i \in \mathcal{N}} \beta_{i}^{2} \mathcal{A}^{2}}\right) .
$$

The gap is maximum if $\sum_{i=1}^{N} \beta_{i}^{2}$ is minimum, subject to $\sum_{i \in \mathcal{N}} \beta_{i}=1$. This is achieved if $\beta_{i}=\frac{1}{N}$ for all $i \in \mathcal{N}$. Thus $\Delta \leq \frac{1}{2} \log \left(\frac{\sigma_{N}^{2}+c \mathcal{A}^{2}}{\sigma_{N}^{2}+\frac{1}{9 N} \alpha^{2} \mathcal{A}^{2}}\right)$. At high SNR, this upper bound on the gap can be approximated by $\delta=\log \left(\frac{3 \sqrt{c N}}{\alpha}\right)$. By substituting $c$ by its value and maximizing $\delta$ with respect to $\alpha$, we conclude that $\delta \leq \log \left(\frac{3 \sqrt{e N}}{\sqrt{2 \pi}}\right)=0.68+\frac{1}{2} \log (N)$ which proves Corollary 7 .

\section{REFERENCES}

[1] A. Chaaban, Z. Rezki, and M.-S. Alouini, "On the capacity of the 2user IM-DD optical broadcast channel," in IEEE Globecom Workshop on Optical Wireless Communication, San Diego, CA, Dec. 2015.

[2] M. A. Khalighi and M. Uysal, "Survey on free space optical communications: A communication theory perspective," IEEE Communication Surveys and Tutorials, vol. 16, no. 4, pp. 2231-2258, 4th quarter 2014.

[3] S. Arnon, J. Barry, G. Karagiannidis, R. Schober, and M. Uysal, Eds., Advanced Optical Wireless Communication Systems. Cambridge University Press, 2012.

[4] H. Elgala, R. Mesleh, and H. Haas, "Indoor optical wireless communication: Potential and state-of-the-art," IEEE Comm. Magazine, vol. 49, no. 9, pp. 56-62, Sep. 2011.

[5] S. M. Moser, "Capacity results of an optical intensity channel with inputdependent Gaussian noise," IEEE Trans. on Info. Theory, vol. 58, no. 1 , pp. 207-223, Jan. 2012.

[6] M. A. Kashani and M. Uysal, "Outage performance and diversity gain analysis of free-space optical multi-hop parallel relaying," IEEE/OSA Journal of Optical Communications and Networking, vol. 5, no. 8, pp. 901-909, Aug. 2013.

[7] A. A. Farid and S. Hranilovic, "Diversity gain and outage probability for MIMO free-space optical links with misalignment," IEEE Trans. on Communications, vol. 60, no. 2, pp. 479-487, Feb. 2012.

[8] - "Capacity bounds for wireless optical intensity channels with Gaussian noise," IEEE Trans. on Info. Theory, vol. 56, no. 12, pp. 60666077, Dec. 2010.

[9] A. Lapidoth, S. M. Moser, and M. Wigger, "On the capacity of freespace optical intensity channels," IEEE Trans. on Info. Theory, vol. 55, no. 10, pp. 4449-4461, Oct. 2009.

[10] A. Chaaban, J.-M. Morvan, and M.-S. Alouini, "Free-space optical communications: Capacity bounds, approximations, and a new spherepacking perspective," submitted to IEEE Trans. on Communications (KAUST Technical Report http://hdl.handle.net/10754/552096), Apr. 2015. 
[11] C. E. Shannon, "A mathematical theory of communication," The Bell System Technical Journal, vol. 27, pp. 379-423, 623-656, 1948.

[12] H. Kim, B. Nachman, and A. E. Gamal, "Superposition Coding is Almost Always Optimal for the Poisson Broadcast Channel," in Proc. of IEEE International Symposium on Info. Theory (ISIT), Hong-Kong, July 2015.

[13] J. M. H. Elmirghani and R. A. Cryan, "Hybrid PPM-CDMA systems utilising OOC for indoor wireless infrared communication," Microwave and Opt. Tech. Lett., vol. 8, no. 1, pp. 44-47, Jan 1995.

[14] B. M. Ghaffari, M. Matinfar, and J. A. Salehi, "Wireless optical CDMA LAN: Digital design concepts," IEEE Trans. on Commununications, vol. 56, no. 12, pp. 2145-2155, Dec. 2008.

[15] U. N. Griner and S. Arnon, "Multiuser diffuse indoor wireless infrared communication using equalized synchronous CDMA," IEEE Trans. on Commununications, vol. 54, pp. 1654-1662, Sep. 2006.

[16] H. Marshoud, V. Kapinas, G. Karagiannidis, and S. Muhaidat, "Nonorthogonal multiple access for visible light communications," IEEE Photonics Technology Letters, vol. PP, no. 99, pp. 1-1, 2015.

[17] T. V. Pham and A. T. Pham, "Max-min fairness and sum-rate maximization of MU-VLC local networks," in IEEE Globecom Workshop on Optical Wireless Communication, San Diego, CA, Dec. 2015.

[18] J. Lian and M. Brandt-Pearce, "Distributed power allocation for multiuser MISO indoor visible light communications," in IEEE Global Communications Conference (GLOBECOM), San Diego, CA, Dec. 2015.

[19] T. M. Cover, "Broadcast channels," IEEE Trans. on Info. Theory, vol. IT-18, no. 1, pp. 2-14, Jan. 1972.

[20] T. H. Chan, S. Hranilovic, and F. R. Kschischang, "Capacity-achieving probability measure for conditionally Gaussian channel with bounded input," IEEE Trans. on Info. Theory, vol. 51, no. 6, pp. 2073-2088, Jun. 2005.

[21] P. Bergmans, "A simple converse for broadcast channels with additive white Gaussian noise," IEEE Trans. on Info. Theory, vol. 20, no. 2, pp. 279-280, March 1974.

[22] A. A. Farid and S. Hranilovic, "Channel capacity and non-uniform signalling for free-space optical intensity channels," IEEE Journal on Selected Areas in Communications, vol. 27, no. 9, pp. 1-12, Dec. 2009.

[23] T. Cover and J. Thomas, Elements of Information Theory (Second Edition). John Wiley and Sons, Inc., 2006.

[24] T. M. Cover, "Comments on broadcast channels," IEEE Trans. on Info. Theory, vol. 44, no. 6, pp. 2524-2530, Oct. 1998.

[25] J. A. Thomas, "Feedback can at most double Gaussian multiple access channel capacity," IEEE Trans. on Info. Theory, vol. 33, no. 5, pp. 711716, Sep. 1987.

[26] P. Bergmans, "Random coding theorem for broadcast channels with degraded components," IEEE Trans. on Info. Theory, vol. IT-19, no. 2, pp. 197-207, March 1973.

[27] H. AlQuwaiee, I. S. Ansari, and M.-S. Alouini, "On the performance of free-space optical communication systems over double generalized Gamma channel," IEEE Journal on Selected Areas in Communications, vol. 33, no. 9, pp. 1829-1840, Sept 2015.

[28] H. Kazemi, Z. Mostaani, M. Uysal, and Z. Ghassemlooy, "Outage performance of MIMO FSO systems in Gamma-Gamma fading channels," in Proc. of IEEE 18th European Conference on Network and Optical Communications, Graz, Austria, July 2013, pp. 275-280.

[29] A. García-Zambrana, C. Castillo-Vázquez, and B. Castillo-Vázquez, "On the capacity of FSO links over Gamma-Gamma atmospheric turbulence channels using OOK signaling," EURASIP J. Wirel. Commun. Netw., vol. 2010, no. 64, pp. 1-11, Jan 2010.

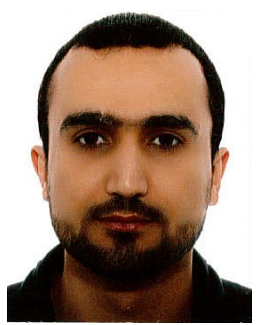

Anas Chaaban (S'09 - M'14) received his Maîtrise ès Sciences degree in electronics from the Lebanese University, Lebanon, in 2006. He received his M.Sc. degree in communications technology and his Dr. Ing. (Ph.D.) degree in Electrical Engineering and Information Technology from the University of Ulm and the Ruhr-University of Bochum, Germany, in 2009 and 2013, respectively. During 2008-2009, he was with the Daimler AG research group on machine vision, Ulm, Germany. He was a Research Assistant with the Emmy- Noether Research Group on Wireless Networks at the University of Ulm, Germany, during 2009-2011, which relocated to Ruhr-Universität Bochum, Germany, in 2011. He was a postdoctoral researcher at the Ruhr-Universität Bochum, Germany, in 20132014, and joined King Abdullah University of Science and Technology as a postdoctoral researcher in 2015 . He received the best poster award at the IEEE Comm. Theory Workshop in 2011, and the best paper award at ICCSPA in 2015. His research interests are in the areas of information theory and wireless communications.

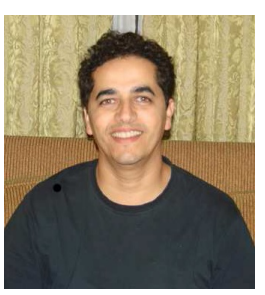

Zouheir Rezki (S'01-M'08-SM'13) was born in Casablanca, Morocco. He received the Diplôme d'Ingénieur degree from the École Nationale de l'Industrie Minérale (ENIM), Rabat, Morocco, in 1994, the M.Eng. degree from École de Technologie Supérieure, Montreal, Québec, Canada, in 2003, and the Ph.D. degree from École Polytechnique, Montreal, Québec, in 2008, all in electrical engineering. From October 2008 to September 2009, he was a postdoctoral research fellow with Data Communications Group, Department of Electrical and Computer Engineering, University of British Columbia. He is now a Senior Research Scientist at King Abdullah University of Science and Technology (KAUST), Thuwal, Mekkah Province, Saudi Arabia. His research interests include: performance limits of communication systems, physicallayer security, cognitive and sensor networks and low-complexity detection algorithms.

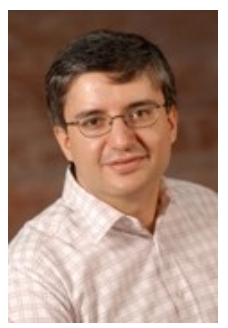

Mohamed-Slim Alouini (S'94, M'98, SM'03, F09) was born in Tunis, Tunisia. He received the Ph.D. degree in Electrical Engineering from the California Institute of Technology (Caltech), Pasadena, CA, USA, in 1998. He served as a faculty member in the University of Minnesota, Minneapolis, MN, USA, then in the Texas A\&M University at Qatar, Education City, Doha, Qatar before joining King Abdullah University of Science and Technology (KAUST), Thuwal, Makkah Province, Saudi Arabia as a Professor of Electrical Engineering in 2009. His current research interests include the modeling, design, and performance analysis of wireless communication systems. 